\title{
Ontogeny and Vulnerabilities of Lapatinib Drug-Tolerant Persisters in HER2+ Breast Cancer
}

Che-wei A. Chang ${ }^{1,2}$, Shaowen Jiang ${ }^{4}$, Azin Sayad ${ }^{2}$, Kevin R. Brown $^{8}$, Allison Nixon ${ }^{8}$, Avantika Dhabaria ${ }^{5}$ ${ }^{6}$, Kwan Ho Tang ${ }^{2,3}$, Jason Moffat ${ }^{8}$, Beatrix Ueberheide ${ }^{5,6}$, Alireza Khodadadi-Jamayran ${ }^{4}$, Aristotelis Tsirigos $^{3,4,7}$, Benjamin G. Neel ${ }^{1,2,3^{*}}$

${ }^{1}$ Department of Medical Biophysics, University of Toronto, Toronto, Ontario, Canada.

${ }^{2}$ Princess Margaret Cancer Centre, University Health Network, Toronto, Ontario, Canada.

${ }^{3}$ Laura and Isaac Perlmutter Cancer Center, NYU Grossman School of Medicine, New York University Langone Medical Center, New York, New York.

${ }^{4}$ Applied Bioinformatics Laboratories, Office of Science and Research, New York University School of Medicine, New York, NY, USA.

${ }^{5}$ Proteomics Laboratory, Division of Advanced Research and Technology, NYU Langone Health, New York, New York.

${ }^{6}$ Department of Biochemistry and Molecular Pharmacology, NYU Langone Health, New York, New York.

${ }^{7}$ Department of Pathology, New York University School of Medicine, New York, NY, USA.

${ }^{8}$ Donnelly Centre, University of Toronto, Toronto, Ontario, Canada.

* Corresponding author and present address: Benjamin G. Neel, NYU Grossman School of Medicine, 522 First Avenue, Smilow Building 12th Floor, Suite 1201, New York, NY 10016. Phone: 212-263-3019; Fax: 212-263-9190; email: Benjamin.Neel@nyulangone.org

Running title (60 characters): Characterization of lapatinib drug-tolerant persisters.

Keywords: HER2-positive breast cancer, tyrosine kinase inhibitor (TKI), resistance, dormancy, quiescence, cancer stem cell, serine/threonine-protein kinase 3 (SGK3)

Competing interests: B.G.N. is a co-founder, holds equity in, and receives consulting fees from Navire Pharmaceuticals and Northern Biologics, Inc. He is a member of the Scientific Advisory Board and receives consulting fees and equity from Avrinas, Inc, and was an expert witness for the Johnson and Johnson ovarian cancer talc litigation in US Federal Court. His spouse has or held equity in Amgen, Inc., Regeneron, Moderna, Inc., Gilead Sciences, Inc., and Arvinas, Inc. J.M. is a shareholder in Northern Biologics and Pionyr Immunotherapeutics and is an advisor and shareholder of Century Therapeutics and Aelian Biotechnology. 


\begin{abstract}
Despite advances in targeted therapeutics, resistance remains a significant clinical problem in HER2-positive (HER2+) breast cancer and can lead to recurrence, metastasis, and death. "Drugtolerant persisters" (DTPs), a sub-population of cancer cells that survive via reversible, nongenetic mechanisms, are implicated in resistance to tyrosine kinase inhibitors (TKIs) in several cancer models, but DTPs following HER2 TKIs exposure have not been characterized extensively. We found that treatment of HER2+ breast cancer lines with lapatinib evoked DTPs with either a luminal-like or a mesenchymal-like transcriptional program and differential sensitivity to lapatinib/anti-estradiol combinations. Lentiviral barcoding and single cell RNA-sequencing showed that luminal-like HER2+ cells cycle stochastically through a "pre-DTP" state, characterized by a $\mathrm{G}_{0}$-like signature, which uniquely gives rise to DTPs upon lapatinib exposure. Lapatinib-DTPs from luminal-like HER2+ cells survive via estrogen receptor-dependent induction of $S G K 3$, thereby rewiring their PI3K/AKT/mTORC1 pathway to enable AKT-independent mTORC1 activation. These results provide new insights into DTP ontogeny and therapeutic vulnerabilities.
\end{abstract}

\title{
STATEMENT OF SIGNIFICANCE
}

DTPs are implicated in resistance to TKIs, other targeted therapies, and some chemotherapies, but their ontogeny and vulnerabilities remain unclear. We find that lapatinibDTPs engage either of two distinct transcriptional programs. At least one type of DTP arises selectively from cells transiting through a $\mathrm{G}_{0}$-like state and exhibits unique vulnerabilities compared with parental HER2+ breast cancer cells. 


\section{INTRODUCTION}

HER2 amplification/over-expression drives $15-20 \%$ of breast cancers, a subset of which co-express estrogen receptor (ER). Depending on tumor size and lymph node status, nonmetastatic HER2-positive (HER2+) and HER2+/ER+ breast cancer patients are first treated by breast-conserving surgery or with neoadjuvant chemotherapy and HER2-directed monoclonal antibodies (trastuzumab plus/minus pertuzumab), followed by surgery $(1,2)$. Surgical patients subsequently receive adjuvant chemotherapy and trastuzumab (+/- pertuzumab). Depending on residual tumor status in patients receiving neoadjuvant therapy, adjuvant trastuzumab and pertuzumab or treatment with the HER2-targeted antibody-drug conjugate trastuzumab emtansine (T-DM1) is recommended. Patients with HER2+/ER+ tumors typically receive anti-estrogen therapy along with the HER2-targeted antibodies. These regimens significantly improve survival rates, compared with chemotherapy alone or trastuzumab and chemotherapy $(1,2)$. Indeed, targeted therapy for non-metastatic HER2+ breast cancer is a major success story in medical oncology, with 5-year disease-free survival of approaching 100\% for node-negative disease and 80-90\% (depending on ER status) for patients with regional lymph node involvement (3).

Metastatic HER2+ breast cancer, by contrast, remains incurable $(1,2)$. Front-line treatment for metastatic HER2+ disease, whether ER+ or ER-, is T-DM1 or chemotherapy and anti-HER2 therapy (Trastuzumab + Pertuzumab). Later options include T-DM1 or HER2-targeted tyrosine kinases inhibitors (TKIs), including lapatinib, neratinib, or tucatinib. The first HER2-directed TKI used clinically, lapatinib, combined with chemotherapy, significantly improves outcome in patients who have progressed on trastuzumab and chemotherapy (4). Yet after an initial response to TKIs, resistance invariably occurs in metastatic HER2+ patients, and ultimately, patients are left without further therapeutic options.

A dual ATP-competitive inhibitor of EGFR and HER2 (5), lapatinib inhibits HER2 extracellular domain (ECD) variants, such as p95HER2, that fail to bind HER2-directed antibodies (6). Antibody-based therapies also might not effectively cross the blood brain barrier (7), so lapatinib has potential benefits for patients with brain metastases. Multiple mechanisms for lapatinib-resistance have been described, including HER3 up-regulation, which increases signaling via HER2/HER3 heterodimers (8-10), activating PIK3CA or loss-of-function PTEN mutations (11), or increased upstream/parallel pro-survival signaling mediated by FAK1, SRC, PRKACA or mTORC1 (12-16). Epigenetic mechanisms for lapatinib resistance involving the mixed lineage leukemia (MLL) complex and bromodomain extra terminal domain (BET) family members also have been proposed $(17,18)$. Although not established by direct experiments, these mechanisms would likely limit the efficacy of other HER2 TKIs.

The above events confer stable resistance to lapatinib, but whether resistant cells pre-exist in HER2+ tumors or emerge during therapy remains unclear. Sharma et al. described a non-genetic, 
drug-tolerant cell state in the EGFR ${ }^{\text {mut }}$ non-small cell lung cancer (NSCLC) line PC9, which could be reversed upon drug withdrawal (19). Such cells, which they termed "drug-tolerant persisters" (DTPs), subsequently were observed in other experimental cancer models, including additional EGFR-mutant NSCLC lines (19), MET-amplified gastric cancer cell lines (20), BRAF-mutant melanoma (21-24), and $A R$-driven prostate cancer $(25,26)$. Upon continued exposure to EGFR inhibitor, Sharma et al. noted that PC9 cells regained the ability to proliferate; these proliferative cells were termed "drug-tolerant expanding persisters" (DTEPs). DTPs (and DTEPs) do not appear to be classical "cancer stem cells," but whether all cancer cells are at any given time equally capable of becoming DTPs remains largely unknown. Although epigenetic modulators (e.g., HDAC inhibitors) that prevent development of DTPs in response to EGFR-TKIs have been identified, the signaling pathways that DTPs employ to survive TKI treatment also are not well understood. Furthermore, DTPs have not been characterized extensively in HER2+ breast cancer.

Here, we provide insights into the ontogeny and potential therapeutic vulnerabilities of lapatinib-DTPs. Our results have potential therapeutic implications, as such cells could serve as a reservoir for the development of stable resistance to lapatinib as well as newer HER2-targeted TKIs. 


\section{RESULTS}

\section{Lapatinib induces DTPs in some, but not all, HER-positive breast cancer cell lines}

We first asked whether HER2+ breast cancer cells exhibited "DTP-like" behavior in response to lapatinib. To this end, we treated ten HER2+ breast cancer lines with $2.5 \mu \mathrm{M}$ lapatinib, which corresponds to average peak plasma levels in patients (27). Three types of response were observed: (I) 3/10 lines were intrinsically resistant and proliferated in the presence of lapatinib; (II) 2/10 showed a cytostatic response; and (III) in 5/10 lines, most cells died after exposure to lapatinib, but a sub-population persisted (Fig. 1A). As noted above, activating PIK3CA mutations or PTEN deletion can confer lapatinib resistance $(11,28)$; notably, Type I and Type II cell lines harbor common "hotspot" activating mutations in PIK3CA (H1047R, E545K) or deletion of PTEN (HCC1569) (29). By contrast, Type III lines (BT474, SKBR3, EFM192A, HCC1419, SUM225) showed behavior similar to that reported for DTPs. Unlike the lines tested by Sharma et al. (19), only EFM192A cells entered a DTEP-like state after 30 days of drug treatment. The other Type III lines (BT474, SKBR3, HCC1419, SUM225) remained dormant for $~ 50$ days (Fig. 1A), a period during which Sharma et al. observed DTEPs emerging from EGFR inhibitor-treated PC9 cells. Type III lines have intact PTEN and normal PTEN expression, although two had rare PIK3CA variants, encoding K111N in BT474 and C420R in EFM192A cells. Both variants have been studied previously; only C420R has increased kinase activity and enhances transformation of chicken embryo fibroblasts $(30,31)$.

Upon drug withdrawal, DTPs (by definition) yield progeny that regain drug sensitivity (19). Similarly, "lapatinib-DTPs" resumed proliferation after lapatinib withdrawal, demonstrating that they were not senescent/permanently growth-arrested. At various times after drug withdrawal, we re-challenged these cells with lapatinib. Consistent with the DTP concept, all Type III category cells that survived initial lapatinib exposure gave rise to lapatinib-sensitive progeny upon drug withdrawal. Individual lines differed in the time that they required for reversion to drug-sensitivity; this interval was highly reproducible across several experiments (Fig. 1B and data not shown). Furthermore, BT474-derived lapatinib-DTPs (BT474-DTPs) were at least as tumorigenic (if not more so) as parental BT474 cells, as assessed by a limiting dilution assay in NOD.CgPrkdc ${ }^{\mathrm{scid}} \mathrm{I} 12 \mathrm{r}^{\mathrm{tm} 1 \mathrm{Wjl}} / \mathrm{SzJ}$ (NOD scid gamma, NSG) mice (Fig. 1C). Hence, these cells not only have the potential to restart proliferation following lapatinib withdrawal, they can also seed new tumors, and conceivably, could contribute to metastasis.

\section{Lapatinib-DTPs display two distinct transcriptional profiles}

We next compared the transcriptomes of lapatinib-DTPs and their parental counterparts by bulk RNA sequencing (RNA-seq). Unsupervised hierarchical clustering segregated the samples into two subgroups, which had transcriptional programs distinct from each other and from their respective parental counterparts (Fig. 2A, Supplementary Tables 1-3). Gene Set Enrichment Analysis (GSEA) revealed that the DTPs in one cluster, hereafter "mesenchymal-like", 
differentially activated genes annotated as "hallmark of epithelial to mesenchymal transition". By contrast, "luminal-like" DTPs were enriched for the "hallmark estrogen response early" gene set (Figs. 2B and 2C). Differentially expressed genes (DEGs) between DTPs and parental cells from the two subgroups also enriched for distinct sets of transcription factor binding sites by Chip Enrichment Analysis (ChEA): DEGs in luminal-like DTPs showed enrichment for ESR1 sites, whereas mesenchymal-like DTPs were enriched for SMAD4 and SOX2 sites (Supplementary Fig. S1A-D).

These findings suggested that when HER2 signaling is abrogated by lapatinib, luminal-like DTPs survive via ESR 1-driven signaling. Consistent with this hypothesis, such lines (BT474 and HCC1419) were killed effectively by lapatinib and fulvestrant (Lap + Ful, Figs. 2D and 2E). Almost all DTPs were eradicated in the former upon combination treatment, as evident by the lack of cell re-growth in Lap+Ful compared with lapatinib alone (Fig. 2F), and response to Lap + Ful correlated with basal and induced ESR1 levels (Fig. 2G). Collectively, these data show that DTPs can arise via two distinct transcriptional programs, which in turn results in differential sensitivity to anti-cancer drugs.

\section{Lapatinib-DTPs are organized stochastically}

To clarify their ontogeny, we asked whether lapatinib-DTPs belong to a pre-existing cellular hierarchy (e.g., a "cancer stem cell" model) or arise stochastically. We transduced a lentiviral barcode library into BT474 cells at a low multiplicity of infection $(\mathrm{MOI}=0.1)$ to ensure that each cell received only one barcode; this approach enabled tracking of up to 100,000 unique clones (Supplementary Fig. S2A and Methods). Infected cells were permitted to expand for approximately six weeks, such that each barcode was represented abundantly in the overall cell population. Approximately $12 \%$ of BT474 cells survive 14 days of lapatinib treatment (Fig. 1A). If HER2+ breast cancer lines maintain a pre-existing, fixed hierarchy of DTPs and non-DTPs, then a similar percentage of barcodes should be retrieved after 14-day lapatinib treatment, compared to the number of barcodes in the initial population $(\mathrm{t}=0)$. By contrast, if lapatinib-DTPs arise stochastically, substantially $>12 \%$ of barcodes should be retrieved (Supplementary Fig. S2A).

In two independent experiments, we retrieved $62 \%$ and $60 \%$ of barcodes, respectively (Supplementary Fig. 2B-D). We noted a consistent reduction ( 25\%) in barcode representation in untreated control (UT) cells cultured for 14 days without lapatinib in both experiments. These "missing barcodes" were poorly represented in the initial population $(\mathrm{t}=0)$, and presumably decreased to undetectable levels after 14 days in culture. Other have also reported stochastic loss of barcodes upon cell passaging (32). Nevertheless, the retention of most barcodes in the starting population after 14 days of lapatinib indicates that nearly all BT474 cells can give rise to lapatinibDTPs. These data support a stochastic model of DTP ontogeny and suggest that over a 6-week period (or less), essentially every BT474 cell or its progeny has the capacity to transit into the drug-tolerant state. 


\section{Parental BT474 cells occupy states with different predilections to become DTPs}

To investigate the dynamics of DTP generation, we performed single-cell RNA sequencing (scRNA-seq) on untreated, 6-hour lapatinib-treated (6h), and 14-day lapatinib-treated BT474 cells (DTPs), and analyzed these data using iCellR (33). We chose the 6-hour point because lapatinibtreated BT474 cells began to die at that time. Supervised analysis of the scRNA-seq results, using the DEGs in BT474-DTPs (BT474-DTP DEG) derived from the bulk RNA-seq (Fig. 2 and Supplementary Table 3), showed that most were induced or suppressed progressively upon lapatinib treatment (Fig. 3A). Closer examination, however, revealed bi-modal expression of genes that ultimately are down-regulated in lapatinib-DTPs ("BT474-DTP Down DEG") in untreated BT474 cells (compare shapes of violin plots of UT cells using BT474-DTP UP and BT474-DTP Down genes, respectively). This finding, combined with the barcoding results described above, suggested that although over time, all BT474 cells have the potential to become DTPs, at any given instant, only select cells exist in a state ("pre-DTPs") conducive to DTP generation.

To explore this intriguing possibility, we performed additional analyses on untreated (UT) BT474 cells. Unsupervised hierarchical clustering identified three major clusters (Fig. 3B), of which cluster B alone displayed significant enrichment for both up- and down-regulated BT474DTP DEGs, compared with the other two (Fig. 3C). Gene ontology (GO) enrichment analysis (using the Enrichr algorithm) showed that the three unsupervised clusters were distinguished largely by genes associated with distinct cell cycle phases (Supplementary Fig S3A; Supplementary Tables 4 and 5): Cluster A showed enrichment for genes involved in the G1/S transition, whereas cluster $\mathrm{C}$ was enriched for $\mathrm{G} 2 / \mathrm{M}$ genes. Additional supervised analysis using annotated cell cycle signatures that distinguish cells in different cell cycle phases, this time including a $\mathrm{G}_{0}$ signature (34), showed strong overlap between cells in the putative "pre-DTP" cluster (cluster B) and $\mathrm{G}_{0}$ cells (Figs. 3D-F). Conversely, most $\mathrm{G}_{0}$ cells, and a few cells annotated as "G1S or MG1", differentially expressed BT474-DTP Up and Down DEGs, as well as luminallike DTP Up and Down DEGs (Fig. 3G; Supplementary Figs. S3B-E). The other luminal-like cell line, HCC1419, also contained a $\mathrm{G}_{0}$ cluster that differentially expressed HCC1419-DTP Up and Down DEGs (Supplementary Table 6) and luminal-like Up and Down DEGs (Figs. 3H and 3I; Supplementary Figs. S3G-L). Collectively, these data demonstrate that, consistent with findings in other cell systems (34,35), randomly proliferating BT474 and HCC1419 cells maintain a significant, but dynamic, population of $\mathrm{G}_{0}$ cells. These untreated $\mathrm{G}_{0}$ cells differentially express a significant percentage of the genes that ultimately distinguish lapatinib-DTPs from parental cells.

In attempt to obtain direct, experimental evidence for the existence of this $\mathrm{G}_{0}$-like "preDTP" state in untreated BT474 cells, we searched for cell surface markers differentially expressed in cluster B (pre-DTPs) compared with the other two clusters. We identified 20 DEGs that were up-regulated in cluster B and in BT474-DTPs, including those encoding surface proteins NPY1R, MAN1A1, ABCC5, CLDN8, PSCA, and PIK3IP1 (Fig. 4A). We focused on NPY1R, which encodes neuropeptide $\mathrm{Y}$ receptor $\mathrm{Y} 1$ and was enriched in the pre-DTP cluster (Supplementary Figs. S4A 
and S4B). Using an available anti-NPY1R antibody, we purified BT474 cells with low, medium, and high levels of NPY1R surface expression by fluorescence-activated cell sorting (FACS) (Supplementary Fig. S4C). Remarkably, untreated BT474 cells with successively higher NPY1R expression (NPY1R $\mathrm{R}^{\mathrm{lo}}<<\mathrm{NPY} 1 \mathrm{R}^{\mathrm{mid}}<\mathrm{NPY} 1 \mathrm{R}^{\text {hi }}$ ) also had successively increased (immediate) resistance to lapatinib (Fig. 4B; Supplementary Fig. S4D). Consistent with the stochastic origin of lapatinib-DTPs as revealed by the barcoding experiments, cells purified based on different levels of surface NPY1R recapitulated heterogeneous NPY1R expression after 14 days in culture (Supplementary Fig. S4E). These fractions also proliferated equally post-sort (Supplementary Fig. $\mathrm{S} 4 \mathrm{~F}$ ), ruling out the possibility that contaminating $\mathrm{NPY} 1 \mathrm{R}^{\text {lo }}$ cells had overtaken the initially NPY1R ${ }^{\text {hi }}$ cell population or vice versa. Bulk RNA-seq revealed $\sim 500$ DEGs in FACS-enriched NPY1R ${ }^{\text {hi }}$ versus NPY1R ${ }^{\text {lo }}$ cells (Supplementary Fig. S5A; Supplementary Table 7), and NPY1R ${ }^{\text {hi }}$ DEGs were enriched significantly for BT474-DTP DEGs (Fig. 4C). Taken together, these results provide strong support for the existence of a "pre-DTP" state in untreated BT474 cells. Moreover, they suggest that lapatinib-DTPs are selected from the pool of $\mathrm{G}_{0}$ cells upon TKI exposure.

As noted above, lapatinib-DTPs from BT474 cells displayed enrichment for the estrogen receptor (ER)-driven transcriptome (Fig. 2C). Notably, GSEA revealed "hallmark of estrogen response early" genes were the most differentially enriched gene set in NPY1R ${ }^{\text {hi }}$, compared with NPY1R ${ }^{\text {lo }}$, cells (Fig. 4D; Supplementary Fig. 5B). Multiple ESR1 target genes were expressed at higher levels in NPY1R ${ }^{\text {hi }}$ than in NPY1R ${ }^{\text {lo }}$ cells, suggesting that these cells have basally higher ER signaling activity prior to lapatinib treatment (Supplementary Fig. S6C). Moreover, TCGA data show that $N P Y 1 R^{\text {hi }}$ tumors express higher levels of ESR1 and ESR1 targets (PGR, GREB1, $S T C 2$ ) than those with low NPY1R expression (Supplementary Fig. S6D). Lapatinib treatment induced NPY1R expression in NPY1R ${ }^{\text {hi }}$ cells to even higher levels, compared with NPY1R ${ }^{\text {mid }}$ and NPY1R ${ }^{\text {lo }}$ cells (Fig. 4E). Intriguingly, NPY1R ${ }^{\text {hi }}$ cells were depleted for genes involved in mitosis or G2/M checkpoint compared with NPY1R ${ }^{\text {lo }}$ cells (Supplementary Fig. S6B). Co-staining with NPY1R antibody and Hoechst 33342 confirmed that NPY1R ${ }^{\text {hi }}$ cells were mainly in $G_{0} / G 1$ whereas NPY1R ${ }^{\text {lo }}$ cells distributed across all cell cycle phases (Figs. 4F and 4G). Taken together, these data indicate that NPY1R expression marks the $\mathrm{G}_{0}$-like, pre-DTP cells that ultimately can become DTPs upon lapatinib exposure.

Using our scRNA-seq data from the UT, 6h, and DTP samples, we used "pseudotime analysis" to infer the trajectory by which parental or 6h-treated BT474 cells become lapatinibDTPs (Fig. 4H). Nearly all DTPs (>90\%) occupy "DTP state 1" (DTP1); a much smaller number are in "DTP state 2" (DTP2). Cells progressively up-regulate NPY1R as they progress from UT to $6 \mathrm{~h}$ treated to DTP cells (Fig. 4I). NPY1R ${ }^{\text {hi }}$ cells mainly follow the DTP1 route, whereas cells with lower NPY1R levels favor the DTP2 route (Fig. 4J). Moreover, consistent with our finding of a $\mathrm{G}_{0}$-like pre-DTP state, BT474 and HCC1419 cells annotated as $\mathrm{G}_{0}$ favor the main (DTP1) route in the pseudotime analysis (Fig. 4K; Supplementary Figs. S5E and S5F). Direct comparison of the levels of genes preferentially expressed in the Pre-DTP/G $\mathrm{G}_{0}$-like cluster in UT BT475 cells after 6 
hours of lapatinib treatment and in the DTP state, however, reveal different patterns of behavior. For example, expression of some cluster $\mathrm{B} / \mathrm{G}_{0}$ genes actually decrease as cells transit to the DTP state, some change little if at all, whereas others are induced (Supplementary tables 8 and 9). These data indicate that some genes thought to be "induced" by lapatinib treatment are actually "selected" by virtue of their being normally expressed in $\mathrm{G}_{0}$ cells (see Discussion). Taken together, our data establish that only those cells that are transiting through $\mathrm{G}_{0}$ (or possibly just entering or leaving that state) are protected from lapatinib and can enter the DTP state (see Discussion).

\section{Lapatinib-DTPs activate mTORC1 via a PI3K-dependent, AKT-independent pathway}

We next investigated the signaling pathways activated in lapatinib-DTPs that enable their survival. Despite continued, complete inhibition of HER2 by lapatinib luminal- and mesenchymallike lapatinib-DTPs showed partial re-activation of both the PI3K/AKT/mTORC1 and ERK/MAPK pathways (Supplementary Fig. S6A). However, treatment with pathway-specific inhibitors showed that survival of lapatinib-DTPs required PI3K, but not MEK, activity (Supplementary Fig. S6B-E). Surprisingly, however, treatment with the pan-PI3K inhibitor BKM120 induced higher cytotoxicity than two AKT inhibitors (GSK690693 and MK-2206) tested over a large dose range; notably, these inhibitors have different mechanisms of inhibition (Figs. 5A and 5B; Supplementary Figs. 7A and 7B). Compared with BKM120, GSK690693 and MK2206 also showed variable and lower inhibition, if any, of phosphorylation of AKT substrates including PRAS40, TSC2, FOXO1, and GSK3 $\beta$ (Figs. 5C and 5D; Supplementary Figs. 7C and 7D). Furthermore, in all DTPs, mTORC1 activity, as inferred by S6K (T389) and S6 (S240/244) phosphorylation, was inhibited to a far greater extent by BKM120 than by either AKT inhibitor. In BT474- and HCC1419-DTPs, phosphorylation of TSC2 on S939 was inhibited by BKM120, but not by GSK690693 or MK-2206 (Figs. 5C and 5D). These data demonstrate that lapatinibDTPs are more dependent on PI3K than on AKT for their survival.

\section{SGK3 is transcriptionally up-regulated by estrogen receptor in luminal-like DTPs}

We next sought to identify the pathway that enabled DTPs to activate mTORC1 in a PI3Kdependent, AKT-independent manner and survive. We first asked whether activation of this putative pathway depended on PDK1, which catalyzes phosphorylation of the activation loop and of multiple members of the AGC kinase family (36). Indeed, pharmacological inhibition of PDK1 with GSK2334470 induced cytotoxicity to a similar extent as BKM120 (Supplementary Fig. S7E and S7F). Furthermore, the cytotoxicity of these inhibitors correlated with their ability to inhibit the phosphorylation of mTORC1 substrates (Supplementary Fig. S6G and S6H).

Based on these data, we hypothesized that a PDK1-dependent, AKT-independent kinase was responsible for mTORC1 activation in luminal-like DTPs. $S G K 3$ is a known estrogen-induced gene in breast cancer, and SGK3 has been implicated in AKT-independent survival $(37,38)$. Notably, $S G K 3$ RNA and its product were up-regulated in luminal-like, but not mesenchymal-like, DTPs (Figs. 6A and 6B), and lapatinib-induced $S G K 3$ expression in BT474 and HCC1419 cells 
was blocked by co-administering fulvestrant (Figs. 6C-F). These results provide further evidence that ESR1 activity is induced in cell lines that give rise to luminal-like DTPs, which in turn leads to increased levels of SGK3.

\section{SGK3 phosphorylates six sites on TSC2 to activate mTORC1 in an AKT-independent manner.}

AKT-catalyzed phosphorylation of TSC2 is critical for growth factor-stimulated mTORC1 activation (39-41). Others have demonstrated that SGK1 can phosphorylate TSC2 to activate mTORC1 $(38,42)$, yet whether SGK3 phosphorylates the same sites as AKT and/or which SGK3evoked phosphorylation sites on TSC2 were responsible for mTORC1 activation was unknown. Notably, recombinant SGK3 phosphorylated TSC2 on AKT substrate motif sites in vitro (Fig. 7A). Seven sites on TSC2 (S939, S981, T993, S1130, S1132, T1462, and S1798) conform to the AKT substrate motif, RXRXXS/T. Mass spectrometric analysis of the in vitro kinase reaction showed that SGK3 catalyzed increased phosphorylation on six of these sites (S939, S981, S1130, S1132, T1462, and S1798), compared with the TSC2-only control (Fig. 7B).

Mutation of two conserved AKT phosphorylation sites on TSC2 (S939A and T1462A; SATA mutant) prevents most growth factor-stimulated mTORClactivation(40), although mutation of additional sites (S981, S1130, 1132) is required to completely ablate mTORC1 activity $(39,41,43)$. To ask if these site(s) are required for SGK3-evoked mTORC1 activation, we cotransfected 293T cells with expression constructs for GST-SGK3 alone or with wild type (WT) TSC2 or various phosphorylation site mutants (Fig. 7C) and assessed SGK3-evoked mTORC1 activation in the presence of the AKT inhibitor MK-2206 by immunoblotting with AKT substrate antibodies. The TSC2-SATA and TSC2-5A mutants showed decreased SGK3-evoked phosphorylation, but mutation of all of the sites phosphorylated by SGK3 in vitro was required to abolish SGK3-induced TSC2 phosphorylation in 293T cells (Fig. 7D). Furthermore, only TSC26A completely inhibited SGK3-evoked mTORC1 activation.

As SGK3 is transcriptionally up-regulated in BT474-DTP and HCC1419-DTP, we asked whether SGK3 mediates AKT-independent survival and mTORC1 activation in these cells. We assessed the effects of 14h, a small molecule inhibitor with in vitro IC50s of $10 \mathrm{nM}$ and $4 \mathrm{nM}$ against SGK1 and SGK3, respectively $(38,44)$. Importantly, 14h inhibits SGK1/3 more potently than AKT1 (38). As expected, AKT inhibitors showed partial cytotoxic effects on BT474-DTPs (Fig. 7E). As a single agent, 14h only slightly inhibited survival, but it showed additive killing when combined with either AKT inhibitor. Nevertheless, 14h/AKT inhibitor combinations still suppressed BT474-DTP survival to a lesser extent than BKM120. Likewise, AKT inhibitors only modestly inhibited HCC1419-DTP cell survival (Fig. 7F), and 14h alone partially suppressed HCC1419-DTP survival. In contrast to its effects on BT474 cells, combined 14h and AKT inhibitor treatment showed similar killing effects as $14 \mathrm{~h}$ alone. Notably, SGK3 is up-regulated by 9-fold in HCC1419-DTPs, compared with the HCC1419 parental cells. Hence, HCC1419-DTP 
might be re-wired to become more dependent on SGK3, and thus less responsive to AKT inhibitors.

We also investigated the inhibition of AKT substrates and mTORC1 activation in response to $14 \mathrm{~h}$ and/or AKT inhibitor treatment. GSK690693 or MK-2206 showed minimal inhibition, if any, on AKT substrate phosphorylation (Fig. 7G and H). SGK3 activity was assessed by the phosphorylation of NDRG1 (T346) (45). Treatment with 14h alone inhibited phosphorylation of PRAS40 (T246), GSK3 $\beta$ (S9), and TSC2 (S939), although these effects were more pronounced in HCC1419-DTP than in BT474-DTP. These biochemical effects were consistent with the greater effect of single agent $14 \mathrm{~h}$ on the viability of HCC1419-DTP than on BT474-DTP (Fig. 7E and F) and with the notion that HCC1419-DTPs showed greater dependency on SGK3. In both cell lines, the combination of $14 \mathrm{~h}$ and AKT inhibitors completely ablated phosphorylation on AKT substrate sites and mTORC1 activation. In concert, these data show that luminal-like DTPs survive lapatinib treatment primarily via ER-induced activation of SGK3, which activates AKT-independent mTORC1 activation and cell survival. 


\section{DISCUSSION}

Most cells in TKI-sensitive cancer cell lines die upon drug exposure, but a small number survive lethal doses in a non-proliferative, DTP state. These cells can acquire additional mutations, ultimately leading to stable drug resistance and disease recurrence $(46,47)$. Also, in most clinical settings, including the treatment of HER2+ breast cancer, drugs are not administered continuously or indefinitely. During "drug holidays", DTPs might revive, proliferate, acquire new mutations, and contribute to disease recurrence (48). Consequently, understanding how DTPs arise and identifying ways to prevent their emergence or kill them could improve disease outcome. We find that when HER2+ breast cancer cells are exposed to lapatinib, two subtypes of lapatinib-DTPs emerge, characterized by distinct transcriptomes, driven by different transcription factors, and distinguishable, but convergent, survival signaling pathways. Consequently, these two DTP subtypes have distinct vulnerabilities. Barcoding experiments show that luminal-like lapatinibDTPs are generated stochastically, but scRNA-seq shows that, at any given time, the overwhelming majority ( $\geq 95 \%$ ) of DTPs emerge from a $\mathrm{G}_{0}$-like, "pre-DTP” state, which manifests selective ESR1 target gene activation (Supplementary Fig. 8A). BT474 pre-DTPs can be purified prospectively from bulk parental cells by FACS for NPY1R and shown to give rise to the majority of lapatinib-DTPs. Pre-DTPs activate a subset of ER target genes, but as luminal pre-DTPs progress to DTPs, ESR 1 activity is induced further and transcriptionally up-regulates $S G K 3$. SGK3 then phosphorylates and inactivates TSC2 to mediate mTORC1 activation and survival. Interestingly, mesenchymal-like DTPs do not induce ESR1, but they also activate mTORC1 in an AKT-independent fashion, although via as yet undefined kinase(s) (Supplementary Fig. 8B). Our results provide new insights into lapatinib-DTP ontogeny and identify potential vulnerabilities for these TKI-resistant cells.

Previous studies showed that at least some HER2+ breast cancer cells can give rise to lapatinib-DTPs, but whether this was a general property of HER2+ lines had remained unclear. In our study, nearly half of the HER2+ lines (Type III lines) examined displayed characteristic DTP behavior. Although DTPs maintained quiescence in the presence of lapatinib, when drug was removed in vitro or upon injection into immune-incompetent mice, they could re-enter the cycle and form tumors. Consequently, lapatinib-DTPs might not only be responsible for minimal residual disease/local recurrence, but conceivably (e.g., during drug holidays), they could seed distant sites and contribute to metastasis.

By contrast, Type I/Type II cell lines harbor PTEN copy number loss or activating hotspot PIK3CA mutations (i.e., E545K or H1047R) and do not die upon lapatinib exposure. The failure of Type I and II lines to yield DTPs is not surprising, as previous studies showed that PI3K pathway activation can cause resistance to HER2-targeted therapies, including trastuzumab and lapatinib $(11,49,50)$. Contrary to Sharma et al.'s initial report on NSCLC, only one of the 5 Type III lines analyzed (EFM192A) gave rise to DTEPs. Subsequent studies demonstrated that at least some PC9 
stocks contain cells with pre-existing mutations that confer resistance to EGFR inhibitor, which give rise to "early resistance", whereas 12-16 weeks of TKI treatment are needed before bona fide epigenetically-determined "late resistance" emerges (46). EFM192A cells harbor a gain-offunction PIK3CA mutation (C420R), which is sufficient to transform chicken embryo fibroblast and NIH3T3 cell lines (30,31). Conceivably, HER2+ cells bearing this mutation have dramatically reduced fitness but can survive and slowly expand in lapatinib. We only maintained our cells for up to 8 weeks, which might have been too short for acquired resistant cells to emerge from the other Type III lines.

Clinically, HER2 tumors are classified as HER2+/ER+ and HER2+ alone. These categories overlap with, but are not identical to, the results of large-scale transcriptional profiling studies. For example, The Cancer Cell Genome Atlas (TGCA) described two HER2+ subtypes, "HER2enriched" and "HER2-luminal" (51). The former display higher expression of RTK (FGFR4, $E G F R, E R B B 2)$ and mesenchymal genes, whereas HER2-luminal tumors express luminal cluster genes (GATA3, BCL2, ESR1) and are enriched for estrogen-response gene sets (52). Our "luminal" and "mesenchymal" lines probably model these two patient subgroups. Importantly, our results show that luminal and mesenchymal lines activate distinct transcriptional programs to give rise to their cognate DTPs. The central role of ESR1 in the luminal-like DTP program is exemplified by the dramatic decrease in DTP generation when luminal lines are treated with lapatinib plus fulvestrant or when cells are grown in charcoal-stripped media to avoid contaminating estrogenic compounds in the serum. Remarkably, scRNA-seq analysis of BT474 cells show that the ESR1 program is even partially "on" in pre-DTPs, conveniently allowing us to enrich for these cells by sorting for an ESR1 target gene NPY1R. Most dramatically, ESR1 drives SGK3 upregulation, which rewires the PI3K/mTORC1 program in luminal-like DTPs. Our results thus provide a mechanistic underpinning for the empirically derived use of anti-estrogen plus HER2+ therapy in HER2+/ER+ breast cancer patients (53). By contrast, mesenchymal-like DTPs appear to use a $\mathrm{SMAD} / \mathrm{SOX}$-driven transcriptional program to escape from lapatinib-induced killing. It will be important to delineate the molecular drivers for this program (e.g., TGF $\beta$, BMPs) so that it can be targeted prospectively in patients with HER2+ER- breast cancer.

Concepts such as dormancy, quiescence, "cancer stem cells", persistence, residual disease and their relationship to eventual disease recurrence and therapeutic resistance have long been recognized and debated (54-57). In part, these debates reflect variable definitions or loose terminology. The classical "cancer stem cell" (CSC) hypothesis, for example, posits a defined hierarchy in which a limited number of CSCs can self-renew and give rise to all other cells in a tumor $(58,59)$. Consequently, CSCs by definition cannot be "induced" or "plastic", yet some studies have described them as such $(60,61)$. CSCs are often described as slow-cycling and more drug-resistant than bulk tumor cells, although such properties are not intrinsic to the CSC concept, which primarily requires that tumors show hierarchical cellular organization. Previous studies of 
other malignancies, using limiting dilution (including Luria Delbruck fluctuation analysis) and/or FACS for surface markers or reporters $(20,22,62)$, have concluded that DTPs arise stochastically. Similarly, our barcoding experiments provide unambiguous evidence that lapatinib-DTPs arise stochastically. Over a several week period, essentially every BT474 cell has the capacity to become a DTP. Therefore, to the extent that DTPs also occur in vivo (a notion supported by studies of other malignancies; see below), drug resistance in HER2+ breast cancer is not likely to be explained by therapy-resistant CSCs.

Yet while every cell has the capacity to become a DTP over time, our scRNA-seq data show that at any given time, only a select fraction of BT474 cells (and, most likely, HCC1419 cells) can become DTPs upon lapatinib exposure. This population (Cluster B in BT474 cells) shows increased or decreased expression of subsets of genes that are up- or down-regulated in "full blown" lapatinib-DTPs. UMAP analysis demonstrates that this "pre-DTP" population shows nearly complete overlap with cells that exhibit a $\mathrm{G}_{0}$-like transcriptional signature, while pseudotime trajectory analysis shows that these $\mathrm{G}_{0}$-like pre-DTPs transit into DTPs when exposed to lapatinib. Most compellingly, NPY1R, a marker identified based on the scRNA-seq analysis, can prospectively enrich for cells with markedly greater propensity to give rise to lapatinib-DTPs.

Our results have similarities to, but also distinctions from, earlier observations on different malignancies. Although it is usually believed that cultured cell lines in complete media conditions cycle continuously and only enter $\mathrm{G}_{0}$ upon growth factor depletion, work by Spencer and Meyer shows that, depending on the level of CDK2 activity at M phase exit, cell either transit through $\mathrm{G}_{0}$ or directly enter G1 (35). The relative fraction of the cell population that enters $\mathrm{G}_{0}$ vs. G1 is cell line-dependent and is a major determinant of doubling time $(35,63)$. Our finding of a significant fraction of untreated BT474 and $\mathrm{HCC} 1419$ cells with a $\mathrm{G}_{0}$-like transcriptome comports with and extends these observations. Similarly, $B R A F^{V 600 E}$ mutant melanoma cell lines (particularly in 3D culture) have a "slow cycling" fraction of cells marked by JARID1B (encoded by KDM5B) expression, which is required for continuous passaging of tumors (62). Similar to our pre-DTPs, JARID1B-positive and -negative cells stochastically interconvert, and treatment with the BRAF inhibitor Vemurafenib enriches for JARID1B+ cells (23). Subsequent studies showed that the same lines stochastically express select RTKs (e.g., EGFR, TRKA, AXL) in the absence of drug exposure, and purifying these RTK+ cells also enriches for resistance for Vemurafenib. Human melanoma samples also have small populations of $K D M 5 B$-positive cells (62) and scRNA-seq (64) shows that $K D M 5 B$ expression correlates with a " $\mathrm{G}_{0} / \mathrm{G} 1$ " signature (which, most likely, is a "G0" signature). Furthermore, a recent report indicates that cells in a $\mathrm{G}_{0}$-like neural crest state pre-exist in a zebrafish melanoma model and are selected for upon BRAF inhibitor treatment (65) While none of these reports explicitly equate $\mathrm{G}_{0}$-like cells with pre-DTPs, in concert the data strongly suggest that this likely to be the case. 
By contrast, other studies, including Sharma et al.'s original report, imply that DTPs arise as a consequence of drug-induced epigenetic changes (66). Our results suggest that these conclusions might require revision. For example, KDM5B and $K D M 6 B$ are upregulated in BT474 pre-DTPs, and they also are "G $\mathrm{G}_{0}$ signature genes" $(34,67)$. However, their expression actually declines as pre-DTPs transit to DTPs (Supplementary Table 8). By contrast, KDM3A and KDM7 are present at higher levels in DTPs than in parental cells and pre-DTPs (Supplementary Table 9). It will be important to determine the extent to which pre-existing epigenetic differences in pre$\mathrm{DTPs} / \mathrm{G}_{0}$ cells are responsible for their ability to convert to DTPs upon lapatinib exposure. Similarly, Hangauer et al. reported that BT474-DTPs globally "down-regulate" antioxidant genes, including genes encoding glutathione peroxidases such as GPX1, GPX2 and GPX4. This observation led them to find that GPX4 inhibition is selectively toxic to DTPs. However, BT474 cell pre-DTPs/Go-like cells also display lower levels of GPX1 and GPX4 compared with other untreated cells (Supplementary Tables 4 and 5). These findings suggest selection of pre-existing GPX1/4-low pre-DTP/G 0 cells, rather than induction of a GPX1/4-low state and could also explain why Hangauer et al. found that pre-treatment with a GPX4 inhibitor prevents lapatinib-DTP generation (66).

Taken together, these observations suggest that defining the vulnerabilities of pre- $\mathrm{DTP} / \mathrm{G}_{0}$ cells could lead to new targets to prevent emergence of resistance in multiple cancer models. Notably, expression of $B A X$, which encodes the eponymous pro-apoptotic BCL2 family member, is lower in BT474 pre-DTPs (Cluster B), compared with other untreated cells (Supplementary Tables 4 and 5). These data could help explain why pre-DTPs are more resistant to lapatinib, and they raise the possibility that combining HER2 TKIs, ER antagonists, and BCL2 inhibitors could have therapeutic benefit. Pre-DTPs also have higher expression of $D Y R K 1 B$, whose gene product is proposed to enforce quiescence (68). Conceivably, DYRK1B inhibition could block occupancy of the $\mathrm{G}_{0}$-like pre-DTP state in luminal-like HER2+ cells, thereby preventing the development of luminal-like DTPs upon lapatinib treatment.

Although some features of lapatinib-DTPs reflect selection based on the pre-existing properties of $\mathrm{G}_{0}$ cells, others are clearly induced upon drug exposure (Supplementary Fig. 8A, Supplementary Tables 4, 5 and 9). Induction of these genes might also reflect intrinsic differences in the wiring diagram of $\mathrm{G}_{0}$ and non- $\mathrm{G}_{0}$ cells, but regardless of the precise mechanism, our data suggest that induced genes can provide targets for therapeutic intervention. For example, BCL2 is induced in BT474 DTPs (Supplementary Table 3) and could be targeted with BH3 agonists (e.g., Venetoclax). Furthermore, HER2+ breast cancer cell lines are typically addicted to PI3K-AKTmTORC1 pathway $(69,70)$. Our results show that lapatinib-DTPs, either luminal- or mesenchymal-like, reactivate PI3K/mTOR signaling via pathway rewiring. Although combining lapatinib and PI3K inhibitors could be limited by toxicity (71), targeting pathway rewiring could prove beneficial. $S G K 3$ is an estrogen receptor target gene $(72,73)$, but less is known about the role of ER-SGK3 axis in cancer. Comporting with previous reports that SGK1 or SGK3 can mediate 
AKT-independent activation of mTORC1 and survival in cancer cells treated with PI3K $\alpha$-specific or AKT inhibitors $(38,42)$, our data implicate $S G K 3$ as a key ER target that mediates survival in response to HER2 inhibition in luminal-like DTPs.

Our study also provides new mechanistic insights into how SGK3 activates mTORC1. Previous studies reported that AKT phosphorylates five sites on TSC2 (S939, S981, S1130, S1132, T1462), and all of these sites were required for AKT-mediated activation of mTORC1 $(39-41,43)$. Our MS analysis demonstrates that SGK3 can phosphorylate the five previously identified sites on TSC2, as well as one additional site (S1798). Furthermore, we find that all six SGK3-evoked sites must be ablated to completely abolish SGK3-mediated mTORC1 activation. Castel et al., also demonstrated that SGK1 phosphorylates 6 sites (S939, S981, S1130, S1132, T1462 and S1798) on TSC2 (42). Our results also contrast with previous work arguing that PRAS40 is a selective AKT target (38); although this might be the case for endogenous levels of SGK3, when SGK3 is upregulated, we find that it can mediate PRAS40 phosphorylation (Fig. 7H). Conversely, we find that NDRG1 (T346) phosphorylation is primarily dependent on SGK3, although we did observe additive inhibition of NDRG1 (T346) phosphorylation when AKT and SGK3 inhibitors were combined consistent with previous reports that both kinases can phosphorylate this site $(42,74,75)$.

In summary, our study shows that two distinct transcriptional programs are elicited in response to lapatinib treatment in the HER2-positive breast cancer cells that give rise to DTPs. Lapatinib-DTPs are generated by selection of a subset of $\mathrm{G}_{0}$ cells that then further induce one of these transcriptional programs to enable survival. For luminal-like DTPs, the induced ESR1dependent program results in re-wiring in the PI3K/AKT/mTORC1 pathway, which can be targeted by combining HER2 TKIs with anti-estrogen therapies and possibly SGK3 inhibition. These findings provide novel insights into DTP ontogeny and vulnerability and should aid the development of new strategies to target luminal-like DTPs. Furthermore, they provide a roadmap for elucidating analogous strategies for mesenchymal-like DTPs as well as DTPs in other malignancies. 


\section{MATERIALS AND METHODS}

\section{Reagents}

Tissue culture reagents, including regular or phenol-free DMEM, RPMI, FBS and Charcoal-stripped FBS, were purchased from Wisent Bioproducts. $\beta$-Estradiol (E2) was a generous gift from Dr. Mathieu Lupien, Princess Margaret Cancer Center, Toronto, ON, Canada. PD0325901 was synthesized in the Neel lab as described (76). 14h was synthesized by BioDuro according to a published protocol (44). Lapatinib ditosylate was purchased from LC Laboratories. Fulvestrant, BKM120, MK2206, and GSK690693 were purchased from Selleck Chemicals. GSK2334470 was purchased from Tocris. Recombinant SGK3 (cat \#14-647) was purchased from EMD-Millipore.

\section{Plasmids and Site-directed Mutagenesis}

The expression construct for $S G K 3-G S T$ was provided by Dr. Alex Toker (Beth Israel Deaconess Medical Center, Boston, MA). pcDNA3-based plasmids encoding FLAG-tagged wild type and SATA (S939A/T1462A)-mutant TSC2 (40) were generated by Dr. Brendan Manning (Harvard School of Public Health, Boston, MA) and were obtained from Addgene. To construct the TSC2-5A (S939A, S981A, S1130A, S1132A, T1462A) and TSC2-6A plasmids (S939A, S981A, S1130A, S1132A, T1462A, S1798A), the TSC2-SATA construct was used as the template for site-directed mutagenesis reaction using the QuikChange Multi Site-Directed Mutagenesis Kit (Agilent Technologies). The sequences of all point mutations were verified by Sanger sequencing.

\section{Cell Culture and Transfections}

All cell lines were purchased from the American Type Culture Collection or Deutsche Sammlung von Mikroorganismen und Zellkulturen. Genotypes of all lines were confirmed by short tandem repeat (STR) analysis (69). Cells were tested regularly for Mycoplasma by using a PCRbased kit from Agilent Technologies. 293T, MDA-MB-453, BT474, SKBR3 and UACC893 cells were maintained in DMEM supplemented with 10\% fetal bovine serum (FBS) and penicillin and streptomycin (Pen/Strep). MDA-MB-361 cells were maintained in DMEM, supplemented with 20\% FBS and Pen/Strep. HCC1569, HCC202, HCC1419 and EFM192A cells were maintained in RPMI supplemented with 10\% FBS and Pen/Strep. SUM225 cells were maintained in Ham's F12, supplemented with 5\% FBS, $5 \mu \mathrm{g} / \mathrm{ml}$ insulin, $1 \mu \mathrm{g} / \mathrm{ml}$ hydrocortisone, and $10 \mathrm{mM}$ HEPES. DMEM, RPMI, and FBS were purchased from WISENT Bioproducts. Transient transfections were performed with LipoD293 ${ }^{\mathrm{TM}}$ In Vitro DNA Transfection Reagent (SignaGen Laboratories), according to the manufacturer's instructions.

\section{Cell Proliferation and Regrowth Assays}

HER2+ breast cancer lines were exposed to $2.5 \mu \mathrm{M}$ lapatinib, and viable cell number was quantified at various times by using a Vi-Cell counter (Beckman-Coulter). Viable cell numbers at each time point were normalized to the viable cell count before drug exposure to calculate \% survival relative to $\mathrm{t}=0$. To assess the reversibility of lapatinib tolerance, cells were trypsinized, 
washed three times in PBS, and then re-plated in standard growth media without lapatinib. At the indicated times after drug withdrawal, cells were trypsinized and re-plated on 6-well plates at 500,000-1,000,000 cells/well. Cells were allowed to attach overnight, some wells were trypsinized to obtain an initial viable cell count $\left(\right.$ Count $\left._{t}\right)$, and the rest were treated with lapatinib for 7 days to obtain the day 7 viable cell count (Count ${ }_{\mathrm{t}}$ ). Survival of cells following drug re-challenge was calculated as Count $_{\mathrm{t}} / \mathrm{Count}_{\mathrm{t} 0}$.

For assessing the response of parental cells to lapatinib in combination with fulvestrant or estrogen depletion, cells (10,000/well) were seeded into 96-well plates. NPY1R-selected cells were treated immediately after FACS with lapatinib and/or the indicated treatments for 14 days and then switched to the regular growth media without the drug for 14 days to assay for regrowth. Residual cells were imaged by using an IncuCyte apparatus with a colored mask used to show cells that remained on the plate.

The cytotoxic effects of various drugs were assessed by treating DTPs in 96-well plates (1,000-3,000 cells/well) with serial dilutions of each agent for 96 hours. At the end-point of the assay, cell number was estimated by using AlamarBlue ${ }^{\circledR}$ Cell Viability Reagent (Life Technologies) and measuring fluorescence (excitation: $540 \mathrm{~nm}$; emission: $590 \mathrm{~nm}$ ) with a Spectramax microplate reader (Molecular Devices).

\section{Tumorigenicity Assays}

The indicated number of parental BT474 cells and BT474-DTPs in 1:1 HBSS:Matrigel (BD Biosciences) were injected into the mammary fat pads of NSG mice. Mice were monitored for tumor formation for up to 5 months.

\section{Lentiviral Barcoding}

DNA barcode library: Oligonucleotides comprising a 12 base pair degenerate region (the barcode) followed by two stable bases ( $\mathrm{C}$ or $\mathrm{G}$ ), and one of several four base pair library codes, were synthesized with common flanking regions (Sigma Aldrich, St. Louis, MO, USA). Nested PCR using the common regions generated double-stranded DNA, which was subsequently ligated into a second-generation lentiviral vector ( $\mathrm{LLJM1}$ ) containing puromycin resistance cassette and ZsGreen fluorescent marker. Three barcode libraries, each identifiable by a unique library code, were cloned, transformed into E. coli and plated as a pool on solid media. More than $5 \times 10^{6}$ bacterial colonies were scraped and pooled for two of the high diversity libraries. Plasmid DNA was maxi-prepped, and a sample was sequenced to confirm diversity of $>10^{6}$ unique barcodes. These libraries were named Library 0 and Library 1 . The third library was generated for use as standard spike-in controls. Single colonies were selected, prepped and Sanger sequenced to identify several standard barcodes.

Lentiviral Transduction and Barcode Experiments: Lentiviruses containing barcode plasmid libraries were produced in HEK293T cells (HEK 293T/17, ATCC CRL11268). BT474 cells $\left(1 \times 10^{6}\right)$ were plated on a $6 \mathrm{~cm}$ dish and infected with the lentiviral library at $\mathrm{MOI}=0.1$ to ensure that each barcode was present only in a single cell. The infected population was expanded 
to 50 million cells, and cells were not discarded during passaging to preserve representation of the barcodes. One million cells were analyzed in triplicate to assess the initial representation of the barcodes. Ten million cells were plated in triplicate on $15 \mathrm{~cm}$ dishes, and the cells were treated with $2.5 \mu \mathrm{M}$ lapatinib for 14 days. All remaining cells that were not used for the first experiment were kept in culture until the start of second experiment at day 14. After 14 days of lapatinib treatment, the remaining cells (approximately 1 million cells) were collected for genomic DNA extraction. The experiment was repeated at day 14 to assess if the same barcodes were retrieved in both experiments.

DNA barcode amplification and sequencing: Genomic DNA (gDNA) for all samples was standardized to $400 \mathrm{ng} / \mathrm{uL}$ in nuclease-free water. Sequencing libraries were constructed by PCR amplification using a common 3' primer 'BL Seq Amp 3': AATGATACGGCGACCACCGAGATCT and one of 166 unique 5' primers 'BL Seq Amp 5' XXX”:CAAGCAGAAGACGGCATACGAGATNNNNNNCGATTAGTGAACGGATCTCGA CGGT, where the "N"s represent a unique sample index. Each gDNA sample was amplified in technical triplicate with unique indexes using ExTaq (Takara Cat\#RR001A) with PCR program of: $95^{\circ} \mathrm{C}$ for 5 minutes, $94^{\circ} \mathrm{C}$ for 30 seconds, $65^{\circ} \mathrm{C}$ for 30 seconds, $72^{\circ} \mathrm{C}$ for 30 seconds and back to step $2,32 \mathrm{x}$ followed by a 5 minute hold at $72^{\circ} \mathrm{C}$. PCR efficiency was assessed by running the product on a 3\% agarose gel. The $137 \mathrm{bp}$ barcode library band was quantified using Bio Rad Image Lab software. Equal amounts of each PCR product were pooled into batches and purified using 15\% TBE PAGE gels (Novex). Purified PCR products were quantified using a Qubit, pooled, and sequenced on an Illumina HiSeq2500 with version 4 chemistry using Illumina sequencing primer: ACACTCTTTCCCTACACGACGCTCTTCCGATCT and custom primer: ATCGATACCGTCGAGATCCGTTCACTAATCG for multiplexed sample ID. Samples were de-multiplexed and barcode abundances were analyzed.

Barcode Processing and Analysis: FASTQ files for each sequenced sample were processed using a bespoke Perl script. Each read was examined to identify one of the three expected library codes (CCAA, ACGT, or TGGA) followed by eight bases corresponding to the vector sequence (eg: ATCGATAC), allowing up to one mismatched base for each feature. Reads lacking both of these sequences were discarded. The nucleotide sequence corresponding to the barcode was then extracted as the 18 nucleotides preceding the vector sequence, and all unique barcodes were counted. All barcode count files, one per sample, were then merged into a single matrix. Noise introduced through sequencing or PCR errors was reduced by collapsing barcodes within a Hamming distance of two into a single barcode record, where the barcode with the highest average abundance was retained as the "parent" barcode. Next, samples with fewer than 100,000 filtered sequence reads were removed, and each sample was normalized for sequencing depth by dividing all read counts in a given sample by the sum of all read counts. Technical replicates were combined by averaging, and barcodes that were observed in only one sample were removed as potential artefacts. The final barcode matrix comprised 154,262 barcode sequences. 


\section{Bulk RNA-seq:}

Bulk RNA sequencing was conducted at the Princess Margaret Genomics Center. For RNA extraction, cells were seeded onto 6-well plates at 500,000 cells/well. RNA was extracted by using the RNeasy Mini Kit, followed by treatment with RNase-free, DNase to remove contaminating genomic DNA. RNA (200 ng) was reverse transcribed by using the Illumina TruSeq Stranded Total mRNA kit. Libraries were sized on an Agilent Bioanalyzer, and their concentrations were validated by qPCR. The libraries were then loaded onto an Illumina NextSeq cartridge V2 for cluster generation, and the flow cell was subjected paired-end sequencing on Illumina Nextseq500.

Genomic alignment was performed with STAR v.2.5.2, and reads were mapped to the human reference genome (hg38). Mapped reads were quantified by RSEM v.1.3.0, and differential expression was assessed by using DESeq2. DEGs were identified by a $2 \times 2$ analysis that compared genes expressed in DTP and parental cells after separating lines into luminal-like (BT474 and HCC1419) and mesenchymal-like (EFM192A and SKBR3) groups. ChIP Enrichment Analysis (ChEA) was performed with Enrichr. Pathway and gene ontology analysis were performed by ranking genes according to fold-change in the $2 \times 2$ or the $4 \times 4$ analysis using Bader lab pathway gene sets (Human_GOBP_AllPathways_no_GO_iea_August_01_2018_symbol.gmt). To compare the overlap of DEGs between DTPs and NPY1R ${ }^{\text {hi }}$ cells, the cut-off was set at abs FC $>2$ and $p<0.05$. Statistical enrichment was then assessed by calculating the Fisher's exact test. To ask if NPY1R ${ }^{\text {hi }}$ breast cancers are enriched for ER targets, transcript enrichment was assessed by using cBioPortal and TCGA human breast cancer datasets (51).

\section{Single Cell RNA-sequencing}

Samples were prepared by the Princess Margaret Genomic Centre following the 10X Genomics Single Cell 3' Reagent Kits v2 user guide. Briefly, samples were washed two times in PBS (Life Technologies) + 0.04\% BSA (Sigma), re-suspended in PBS $+0.04 \%$ BSA, and loaded onto a 10X single cell A chip. After droplet generation, samples were transferred onto pre-chilled 96-well plates (Eppendorf), heat-sealed, and incubated overnight in a Veriti 96-well thermal cycler (Thermo Fisher) for reverse transcription (RT). Following RT, cDNA was recovered using the Recovery Agent provided by $10 \mathrm{X}$, and subsequently purified by using Silane DynaBead (Thermo Fisher) mix, as outlined by the user guide. Purified cDNA was amplified for 13 cycles before purification on SPRIselect beads (Beckman). Samples were diluted 4:1 (elution buffer (Qiagen):cDNA), and cDNA concentration was determined with a Bioanalyzer (Agilent Technologies). cDNA libraries were prepared as outlined by the Single Cell 3' Reagent Kits v2 user guide with modifications to the PCR cycles based on the calculated cDNA concentration.

The molarity of each library was calculated based on library size, as measured by a Bioanalyzer and qPCR quantification (Roche/Kapa BioSystems). Samples were pooled and normalized to $10 \mathrm{nM}$, then diluted to $2 \mathrm{nM}$ using elution buffer (Qiagen) containing $0.1 \%$ Tween20 (Sigma). Each $2 \mathrm{nM}$ pool was denatured in $0.1 \mathrm{~N} \mathrm{NaOH}$ at equal volumes for 5 minutes at room temperature. Library pools were further diluted to $20 \mathrm{pM}$ using HT-1 (Illumina), before dilution to a final loading concentration of $16 \mathrm{pM}$, and $100 \mu \mathrm{l}$ from the $16 \mathrm{pM}$ pool were loaded into each 
well of an 8-well strip tube and placed onto a cBot (Illumina) for cluster generation. Samples were sequenced on a HiSeq 2500 V4 with the following run parameters: Read $1-26$ cycles, read $2-$ 98 cycles, index $1-8$ cycles.

\section{scRNA-seq Informatic Analysis}

Pre-processing: After confirming cDNA integrity, library quality, number of cells sequenced, and mean number of reads per cell, we used Cell Ranger Single-Cell Software (https://support.10xgenomics.com/single-cell-gene-expression/software/pipelines/latest/what-iscell-ranger) to map the reads and generate gene-cell matrices. A quality control was then performed on the cells to calculate the number of genes, UMIs, and the proportion of mitochondrial genes for each cell using the iCellR R package (v0.99.0) (https://github.com/rezakj/iCellR). A similar workflow was used to pre-process the BT474 and HCC1419 cell samples.

Cells with particularly low or high numbers of covered genes (BT474: min. genes=200, max. genes=7500; HCC1419: min. genes=1250 max. genes=7500) and/or high mitochondrial counts (BT474: max.mito=0.30; HCC1419: $\max \cdot \operatorname{mito}=0.20$ ) were filtered. The matrix was normalized based on top 500 ranked genes (top 500 genes sorted by base mean) geometric library size factor (ranked glsf, top.rank $=500$ ) using iCellR. This approach reduces the effect of dropouts (non-zero events counted as zero) in normalization by only considering the highly expressed genes. A general gene statistic was then performed to calculate gene dispersion, base mean, and cell coverage to use to build a gene model for Principal Component Analysis (PCA). A second round of PCA was performed based on the top 20 and bottom 20 genes predicted in the first 10 dimensions of PCA to fine-tune the results.

Cell Cycle Analysis: We used the cell cycle phase-specific gene signatures defined by Xue et al. (34) to calculate phase-specific scores for $\mathrm{G}_{0}, \mathrm{G} 1 \mathrm{~S}, \mathrm{G} 2 \mathrm{M}, \mathrm{M}, \mathrm{MG} 1$, and $\mathrm{S}$. To account for differences in gene set sizes, we used a scoring method similar to that described by Tirosh et al. (64). For each cell, we compared the sum of phase-specific gene expression (log 10 transformed UMIs) to the distribution of random background gene sets, where the number of background genes is identical to the phase-specific gene set and are drawn from the same expression bins. Each cell was then assigned to a cell-cycle stage based on the highest phase-specific score. Cell-cycle UMAP was performed by employing Seurat (v3.1.0), using all of the cell-cycle-phase-specific geneexpression signatures as the input features in the RunUMAP function (umap.method = "umaplearn", metric = "correlation").

Individual Sample Analysis: After pre-processing of the scRNA-seq data, we selected the first 15 dimensions of PCA for each time-point sample (untreated, 6h-treated, DTP) of BT474 and HCC1419 cells for additional clustering and dimensionality reduction. Clustering was performed using iCellR with the following settings: iCellR options: clust.method = "ward.D", dist.method = "euclidean", index.method = "kl"; phonograph options: $\mathrm{k}=200$, dims $=1: 15$ on these principal components, and dimension reduction was accomplished by using Uniform Manifold Approximation and Projection (UMAP) (77). For scaled normalized gene set expression, 
$\log 2$ (normalized counts +1 ) gene were calculated for each expressed gene, and then center scaling was performed for each gene across the population. Values shown in the box plots represent the sum of the entire gene set expression for each cell. DTP gene expression signatures scores were calculated by selecting the top 150 most significant differentially expressed genes from bulk RNAseq and applying the approach for calculating the cell-cycle-phase specific score described above.

Trajectory Analysis: For each cell line, we used Monocle2 (v2.16.0) to define pseudotime trajectories. The raw count matrix was used as tge input, and the first 10 PCA dimensions of genes identified by iCellR were used for building the trajectories. The reduceDimension function with "DDRTree" algorithm was used to build the trajectory. Cells in the same branch of the trajectory share similar gene expression patterns.

Visualization and Statistical Analysis: All visualization and statistical analyses were performed in R (v4.0.0) (http://www.r-project.org/). Two groups were compared using an unpaired t-test. Significance was defined as ns $\mathrm{P}>0.05$, * $\mathrm{P}<0.05$, ** $\mathrm{P}<0.01, * * * \mathrm{P}<0.0001$, **** $\mathrm{P}<$ 0.0001 . Heat maps were generated by using the pheatmap package (v1.0.12) with scale $=$ "row" argument.

\section{Quantitative RT-PCR}

RNA was extracted using the RNeasy Mini Kit (Qiagen), mRNAs were reverse transcribed with SuperScript III First Strand Synthesis (Invitrogen), and $\mathrm{iQ}^{\mathrm{TM}}$ SYBR ${ }^{\circledR}$ Green Supermix was used for qPCR on CFX96 (Bio-Rad). Relative expression was calculated with the delta-delta $\mathrm{Ct}$ method. Briefly, the $\mathrm{Ct}$ value of the gene of interest is subtracted with $\mathrm{Ct}$ value of the housekeeping gene TBP to yield $\Delta \mathrm{Ct}$ and the $\Delta \mathrm{Ct}$ of from the sample of interest is subtracted with control sample to yield $\Delta \Delta \mathrm{Ct}$. The relative expression is expressed as $2^{-\Delta \Delta \mathrm{Ct}}$. The following primers used in the qPCR: SGK3: F, 5'-GTGCCCGAAGGTTGCATGAT-3' and R, 5'ATCCCTCAAGAGCACACCAA-3'; NPY1R: F, 5'- GCAGGAGAAATACCAGCGGA-3' and R, 5' - TCCCTTGAACTGAACAATCCTCTT-3'; TBP: F, 5'-TGTGCACAGGAGCCAAGAGT3' and R, 5'-ATTTTCTTGCTGCCAGTCTGG-3'.

\section{Flow Cytometry and Fluorescence Activated Cell Sorting (FACS)}

Cells were released from plates by incubating with $0.05 \%$ trypsin until cells detached and stained with primary antibody ( $1 \mu \mathrm{g} / 10^{6}$ live cells) against NPY1R (MAB6400; R\&D Systems) followed by secondary antibody against human $\mathrm{IgG}$ for 30 minutes on ice. Flow cytometry was conducted on a Becton Dickinson analyzer, and FACS was performed by using a FACSAriaII. Post-FACS purity checks were performed to ensure that each fraction was $>90 \%$ of the population of interest. Where indicated, RT-qPCR was conducted to determine whether the sorted fractions showed the expected differential expression of NPY1R. For NPY1R and Hoechst33342 costaining, NPY1R staining was first performed on live cells on ice as above, then cells were fixed 
with 4\% PFA, and permeabilized in $0.1 \%$ Triton-X. Finally, Hoechst33342 $(10 \mu \mathrm{g} / \mathrm{ml})$ was added for 20 minutes, and cells were analyzed on the flow cytometer.

\section{Biochemical Assays}

Immunoblotting: Cells were lysed in RIPA buffer $(50 \mathrm{mM}$ Tris- $\mathrm{HCl}, \mathrm{pH} 7.5,150 \mathrm{mM}$ $\mathrm{NaCl}, 2 \mathrm{mM}$ EDTA, 1\% NP-40, 0.5\% Na deoxycholate, and 0.1\% SDS) with protease and phosphatase inhibitors ( $40 \mu \mathrm{g} / \mathrm{ml} \mathrm{PMSF}, 20 \mathrm{mM} \mathrm{NaF}, 1 \mathrm{mM} \mathrm{Na}_{3} \mathrm{VO}_{4}, 10 \mathrm{mM} \beta$-glycerophosphate, $10 \mathrm{mM}$ sodium pyrophosphate, $2 \mu \mathrm{g} / \mathrm{ml}$ antipain, $2 \mu \mathrm{g} / \mathrm{ml}$ pepstatin $\mathrm{A}, 20 \mu \mathrm{g} / \mathrm{ml}$ leupeptin, and 20 $\mu \mathrm{g} / \mathrm{ml}$ aprotinin). Lysates were cleared by centrifugation at $15,000 \mathrm{~g}$ for 10 minutes at $4{ }^{\circ} \mathrm{C}$, and supernatants were collected. Protein concentrations were quantified by Bradford assay (Thermo Scientific), and 10-30 $\mu$ g total cellular protein were resolved on SDS-PAGE and transferred onto Immobilon-FL PVDF membranes (Millipore). Bound antibodies were detected with IRDye infrared secondary antibodies using the Odyssey Infrared Imaging System (LI-COR Biosciences). Primary antibodies used for immunoblots included: pHER2 Y1221/1222 (2249; Cell Signaling Technology), c-ErbB2 (OP15L; Millipore), pEGFR Y1068 (2234, Cell Signaling Technology), EGFR (2232, Cell Signaling Technology), pERBB3 Y1289 (4791; Cell Signaling Technology), ERBB3 (sc-285, Santa Cruz Biotechnology), pAKT S473 (4060, Cell Signaling Technology), pPRAS40 T246 (13175; Cell Signaling Technology), p-p70 S6 Kinase T389 (9205; Cell Signaling Technology), p-p44/42 (9101L; Cell Signaling Technology), ERK2 (C-18; Santa Cruz Biotechnology Inc.), p-GSK3 $\beta$ S9 (9336; Cell Signaling Technology), pTSC2 S939 (ab52962; Abcam), pS6 S240/244 (2215; Cell Signaling Technology). pFoxO1 (Thr24)/FoxO3a (Thr32) (9464; Cell Signaling Technology), SGK3 (8156; Cell Signaling Technology), pNDRG1 (T346) (5482; Cell Signaling Technology), p-AKT substrate (9614L, Cell Signaling Technology), TSC2 (3990; Cell Signaling Technology).

Kinase Assays: To enrich for dephosphorylated TSC2 to use as a substrate for in vitro kinase assays, pcDNA3-wtTSC2 was transfected into 293T cells, and after 24 hours, transfected cells were treated with $1 \mu \mathrm{M}$ BKM 120 for 1 hour before lysis in kinase assay lysis buffer $(50 \mathrm{mM}$ Tris-HCl, pH 7.5, 150 mM NaCl, 1\% NP-40, 1 mM EDTA, 1 mM EGTA, 40 gg/ml PMSF, 20 $\mathrm{mM} \mathrm{NaF}, 1 \mathrm{mM} \mathrm{Na} \mathrm{VO}_{4}, 10 \mathrm{mM} \beta$-glycerophosphate, $10 \mathrm{mM}$ sodium pyrophosphate, $2 \mu \mathrm{g} / \mathrm{ml}$ antipain, $2 \mu \mathrm{g} / \mathrm{ml}$ pepstatin A, $20 \mu \mathrm{g} / \mathrm{ml}$ leupeptin, $20 \mu \mathrm{g} / \mathrm{ml}$ aprotinin, $0.1 \%$ (v/v) 2mercaptoethanol). FLAG-tagged TSC2 was immunoprecipitated with ANTI-FLAG ${ }^{\circledR}$ agarose beads (Sigma) for 2 hours at $4^{\circ} \mathrm{C}$. Beads were subsequently washed once with high-salt kinase assay wash buffer (50 mM Tris-HCl, pH7.5, $500 \mathrm{mM} \mathrm{NaCl}, 1 \%$ NP-40, $1 \mathrm{mM}$ EDTA, $1 \mathrm{mM}$ EGTA, $40 \mu \mathrm{g} / \mathrm{ml}$ PMSF, $20 \mathrm{mM} \mathrm{NaF}, 1 \mathrm{mM} \mathrm{Na}_{3} \mathrm{VO}_{4}, 10 \mathrm{mM} \beta$-glycerophosphate, $10 \mathrm{mM}$ sodium pyrophosphate, $2 \mu \mathrm{g} / \mathrm{ml}$ antipain, $2 \mu \mathrm{g} / \mathrm{ml}$ pepstatin $\mathrm{A}, 20 \mu \mathrm{g} / \mathrm{ml}$ leupeptin, $20 \mu \mathrm{g} / \mathrm{ml}$ aprotinin, $0.1 \%(\mathrm{v} / \mathrm{v})$ 2-mercaptoethanol), once with kinase assay lysis buffer, and twice with kinase assay buffer (50 mM Tris- $\mathrm{HCl} \mathrm{pH} 7.5,10 \mathrm{mM} \mathrm{MgCl}, 0.1 \mathrm{mM}$ EGTA). Kinase reactions contained recombinant SGK3 (100 ng), $100 \mu \mathrm{M}$ ATP, 0.1\% (vol/vol) 2-mercaptoethanol, $20 \mathrm{mM} \mathrm{NaF}, 1 \mathrm{mM}$ $\mathrm{Na}_{3} \mathrm{VO}_{4}, 10 \mathrm{mM} \beta$-glycerophosphate, and $10 \mathrm{mM}$ sodium pyrophosphate. Reactions were allowed 
to proceed for 30 minutes at $30^{\circ} \mathrm{C}$, and then were terminated by boiling in Laemmli buffer. Proteins from these assays were resolved with $6-10 \%$ SDS-PAGE.

To monitor SGK3 phosphorylation of TSC2 in vivo, 293T cells on $60 \mathrm{~mm}$ dishes were cotransfected with $1.5 \mu \mathrm{g}$ GST-SGK3 and $1.5 \mu \mathrm{g}$ flagged-tagged TSC2 (wildtype TSC2, TSC2SATA, TSC2-5A or TSC2-6A). Cells were allowed to recover in regular growth media for 24 hours, and then were lysed in kinase assay lysis buffer. TSC2 was immunoprecipitated with ANTI$\mathrm{FLAG}^{\circledR}$ beads and subjected to immunoblotting with pAKT substrate antibody. Whole cell lysates and immunoprecipitated TSC2 were resolved by 6-15\% SDS-PAGE.

\section{Mass Spectrometry}

Scaled-up kinase reaction mixtures were resolved by SDS-PAGE. Gels were stained with Coomassie Brilliant Blue R-250 Staining Solution (BioRad), and a band corresponding to FLAGTSC2 was excised and submitted to the Perlmutter Cancer Center Proteomics shared resource at NYU School of Medicine (New York, NY, USA). Bands were cut into $1 \mathrm{~mm}^{3}$ pieces and destained for 15 minutes in a $1: 1(\mathrm{v} / \mathrm{v})$ solution of methanol/100mM ammonium bicarbonate. The buffer was exchanged, and the samples were de-stained for another 15 minutes. This process was repeated for another 3 cycles. Gel pieces were then dehydrated by washing in acetonitrile, dried in a SpeedVac for 20 minutes, reduced in $100 \mu 1$ of $0.02 \mathrm{M}$ dithiothreitol (Sigma) $/ \mathrm{pH} 7.5$ for one hour at $57{ }^{\circ} \mathrm{C}$, and alkylated with $100 \mu \mathrm{l}$ of $0.05 \mathrm{M}$ iodoacetamide (Sigma) for 45 minutes at room temperature in the dark. Gel pieces were once again dehydrated by washing in acetonitrile, and then dried in a SpeedVac for 30 minutes. Sequencing grade modified trypsin (500 ng, Promega) was added directly to the dried gel pieces, followed by enough $100 \mathrm{mM}$ ammonium bicarbonate to cover them. The gel plugs were agitated at room temperature, and digestion was allowed to proceed overnight. Reactions were halted by adding a slurry of R2 $50 \mu \mathrm{m}$ Poros beads (Applied Biosystems) in 5\% formic acid/0.2\% trifluoroacetic acid (TFA) to each sample at a volume equal to that of the ammonium bicarbonate added for digestion. Samples were allowed to shake at $4^{\circ} \mathrm{C}$ for 120 mins, and the beads were loaded onto C18 ziptips (Millipore) equilibrated with $0.1 \%$ TFA by centrifugation for $30 \mathrm{~s}$ at $6,000 \mathrm{rpm}$ in a microfuge. The beads were then washed with $0.5 \%$ acetic acid, and peptides were eluted in $40 \%$ acetonitrile in $0.5 \%$ acetic acid, followed by $80 \%$ acetonitrile in $0.5 \%$ acetic acid. The organic solvent was removed by using a SpeedVac, and the sample was reconstituted in $0.5 \%$ acetic acid.

An aliquot of each sample was loaded onto an Acclaim PepMap trap column (2 cm x 75 $\mu \mathrm{m})$ in line with an EASY-Spray analytical column $(50 \mathrm{~cm}$ x $75 \mu \mathrm{m}$ ID PepMap C18, $2 \mu \mathrm{m}$ bead size) by using the auto-sampler of an EASY-nLC 1200 HPLC (Thermo Fisher Scientific) with solvent A (2\% acetonitrile in $0.5 \%$ acetic acid) and solvent $\mathrm{B}(80 \%$ acetonitrile in $0.5 \%$ acetic acid). Peptides were eluted into an Orbitrap QExactive HF-X Mass Spectrometer (Thermo Fisher Scientific) by using the following gradient: 5-35\% in $120 \mathrm{~min}, 3645 \%$ in $10 \mathrm{~min}$, followed by 45 $100 \%$ in 10 min. High resolution full MS spectra were recorded at a resolution of 45,000, an AGC target of $3 \mathrm{e} 6$, a maximum ion time of $45 \mathrm{~ms}$, and a scan range from 400 to $2000 \mathrm{~m} / \mathrm{z}$. Following each full MS scan, parallel reaction monitoring (PRM) scans were acquired for the peptides of 
interest. MS/MS spectra were collected at a resolution of 15,000, an AGC target of 2e5, maximum ion time of $120 \mathrm{~ms}$, one microscan, $2 \mathrm{~m} / \mathrm{z}$ isolation window, fixed first mass of $150 \mathrm{~m} / \mathrm{z}$, dynamic exclusion of $30 \mathrm{sec}$, and Normalized Collision Energy (NCE) of 27.

MS/MS spectra were searched against the Uniprot human reference proteome database, (downloaded 10/2016) using Byonic software(78,79) (Protein Metrics Inc., San Carlos, CA). The mass tolerance was set to $10 \mathrm{ppm}$ for MS1 and $0.02 \mathrm{Da}$ for MS2 searches. A false discovery rate (FDR) of $1 \%$ was applied at the protein level. The Byonic search included fixed modifications of carbamidomethylation on cysteine and variable modifications of oxidation on methionine, deamidation on asparagine and glutamine, and phosphorylation at serine, threonine and tyrosine residues. The spectra of the peptides of interest were verified manually to localize phosphorylation sites. Relative quantification of the peptides was calculated using Byologic software (Protein Metrics Inc., San Carlos, CA), which uses extracted ion chromatogram areas (XIC areas). The XIC is automated by Byologic from the Byonic search results of potentially identified peptides.

\section{Statistical Analysis}

Data are expressed as mean \pm SEM unless specified otherwise. All statistical analyses were generated by using GraphPad Prism 5. Significance was assessed by two-way ANOVA, one-way ANOVA, or Student's t-test, as appropriate. For ANOVAs, the post-hoc analysis used is indicated in the figure legend. A p-value of less than 0.05 was considered significant.

\section{Data availability}

RNAseq and scRNA-seq data have been deposited in Gene Expression Omnibus (GEO: GSE155342 and GEO: GSE156246). All other reagents are available upon request from B.G.N (Benjamin.neel@nyulangone.org) or J.M. (jmoffat@utoronto.ca).

\section{Code Availability}

The bespoke Perl script for the barcode library analysis is available from J.M. upon request/

\section{ACKNOWLEDGEMENTS}

We thank Neke Ibeh, Zhibin Lu, and Carl Virtanen (Princess Margaret Cancer Center) for bioinformatic analysis of the NPY1R RNA-seq data, and the PCC Genome Technology Center, Applied Bioinformatics Laboratory and Proteomics Laboratory shared resources (P30CA016087) for technical support. We also thank Dr. Alex Toker (Beth Israel Deaconess Medical Center) for providing the GST-SGK3 plasmid. This work was partially supported by NIH grant CA59152 to B.G.N. and CIHR grant MOP-142375 and support from the Ontario Research Excellence Fund to J.M. During part of this study, B.G.N. was a Canada Research Chair, Tier 1 and J.M. holds a Canada Research in Functional Genomics, Tier 1. C.A.C. was supported by Frederick Banting and Charles Best Canada Graduate Scholarship and Doctoral Completion Award from the Department of Medical Biophysics at University of Toronto. 


\section{Author Contributions}

C.A.C., J.M., and B.G.N. designed the study. C.A.C. performed the majority of the experiments. S.J., A.S., K.R.B., A.K. and A.T. performed bioinformatics analyses. A.N. and J.M. performed the DNA barcode amplifications and sequencing. A.D. and B.U. performed the mass spectrometry experiments and analyses. K.H.T. performed the mammary fat-pad injection for the limiting dilution assay. C.A.C. and B.G.N. wrote the manuscript, which all authors helped to edit. 


\section{REFERENCES}

1. Pernas S, Tolaney SM. HER2-positive breast cancer: new therapeutic frontiers and overcoming resistance. Ther Adv Med Oncol. 2019;11:175883591983351.

2. Pondé N, Brandão M, El-Hachem G, Werbrouck E, Piccart M. Treatment of advanced HER2-positive breast cancer: 2018 and beyond. Cancer Treat Rev. Elsevier; 2018;67:1020.

3. Surveillance, Epidemiology and ER (SEER) P. SEER*Stat Data-base: Incidence-SEER 13 Regs Research DATA. Natl. Cancer Institute, Div. Cancer Control Popul. Sci. Surveill. Res. Progr. 2017.

4. Geyer CE, Forster J, Lindquist D, Chan S, Romieu CG, Pienkowski T, et al. Lapatinib plus capecitabine for HER2-positive advanced breast cancer. N Engl J Med. 2006;355:2733-43.

5. Rusnak DW, Lackey K, Affleck K, Wood ER, Alligood KJ, Rhodes N, et al. The effects of the novel, reversible epidermal growth factor receptor/ErbB-2 tyrosine kinase inhibitor, GW2016, on the growth of human normal and tumor-derived cell lines in vitro and in vivo. Mol Cancer Ther. 2001;1:85-94.

6. Scaltriti M, Rojo F, Ocaña A, Anido J, Guzman M, Cortes J, et al. Expression of p95HER2, a truncated form of the HER2 receptor, and response to Anti-HER2 therapies in breast cancer. J Natl Cancer Inst. 2007;99:628-38.

7. Stemmler HJ, Kahlert S, Siekiera W, Untch M, Heinrich B, Heinemann V. Characteristics of patients with brain metastases receiving trastuzumab for HER2 overexpressing metastatic breast cancer. The Breast. 2006;15:219-25.

8. Sergina N V, Rausch M, Wang D, Blair J, Hann B, Shokat KM, et al. Escape from HERfamily tyrosine kinase inhibitor therapy by the kinase-inactive HER3. Nature. 2007;445:437-41.

9. Amin DN, Sergina N, Ahuja D, McMahon M, Blair J a, Wang D, et al. Resiliency and vulnerability in the HER2-HER3 tumorigenic driver. Sci Transl Med. 2010;2:16ra7.

10. Garrett JT, Olivares MG, Rinehart C, Granja-Ingram ND, Sánchez V, Chakrabarty A, et al. Transcriptional and posttranslational up-regulation of HER3 (ErbB3) compensates for inhibition of the HER2 tyrosine kinase. Proc Natl Acad Sci U S A. 2011;108:5021-6.

11. Eichhorn PJ a, Gili M, Scaltriti M, Serra V, Guzman M, Nijkamp W, et al. Phosphatidylinositol 3-kinase hyperactivation results in lapatinib resistance that is reversed by the $\mathrm{mTOR} /$ phosphatidylinositol 3-kinase inhibitor NVP-BEZ235. Cancer Res. 2008;68:9221-30.

12. Schwill M, Tamaskovic R, Gajadhar AS, Kast F, White FM, Plückthun A. Systemic analysis of tyrosine kinase signaling reveals a common adaptive response program in a HER2positive breast cancer. Sci. Signal. 2019.

13. Rexer BN, Ham a-JL, Rinehart C, Hill S, Granja-Ingram NDM, González-Angulo a M, et al. Phosphoproteomic mass spectrometry profiling links Src family kinases to escape from HER2 tyrosine kinase inhibition. Oncogene. 2011;30:4163-74.

14. Moody SE, Schinzel a C, Singh S, Izzo F, Strickland MR, Luo L, et al. PRKACA mediates resistance to HER2-targeted therapy in breast cancer cells and restores anti-apoptotic signaling. Oncogene. Nature Publishing Group; 2014;0:1-11.

15. Brady SW, Zhang J, Tsai MH, Yu D. PI3K-independent mTOR activation promotes lapatinib resistance and IAP expression that can be effectively reversed by mTOR and Hsp90 inhibition. Cancer Biol Ther. 2015;16:402-11.

16. Garcia-Garcia C, Ibrahim YH, Serra V, Calvo MT, Guzman M, Grueso J, et al. Dual 
mTORC1/2 and HER2 Blockade Results in Antitumor Activity in Preclinical Models of Breast Cancer Resistant to Anti-HER2 Therapy. Clin Cancer Res. 2012;18:2603-12.

17. Matkar S, Sharma P, Gao S, Gurung B, Katona BW, Liao J, et al. An Epigenetic Pathway Regulates Sensitivity of Breast Cancer Cells to HER2 Inhibition via FOXO/c-Myc Axis. Cancer Cell. Elsevier Inc.; 2015;28:472-85.

18. Stuhlmiller TJ, Miller SM, Zawistowski JS, Nakamura K, Beltran AS, Duncan JS, et al. Inhibition of Lapatinib-Induced Kinome Reprogramming in ERBB2-Positive Breast Cancer by Targeting BET Family Bromodomains. Cell Rep. The Authors; 2015;1-15.

19. Sharma S V, Lee DY, Li B, Quinlan MP, Takahashi F, Maheswaran S, et al. A chromatinmediated reversible drug-tolerant state in cancer cell subpopulations. Cell. 2010;141:69-80.

20. Raha D, Wilson TR, Peng J, Peterson D, Yue P, Evangelista M, et al. The cancer stem cell marker aldehyde dehydrogenase is required to maintain a drug-tolerant tumor cell subpopulation. Cancer Res. 2014;74:3579-90.

21. Konieczkowski DJ, Johannessen CM, Abudayyeh O, Kim JW, Cooper ZA, Piris A, et al. A melanoma cell state distinction influences sensitivity to MAPK pathway inhibitors. Cancer Discov. 2014;4:816-27.

22. Shaffer SM, Dunagin MC, Torborg SR, Torre EA, Emert B, Krepler C, et al. Rare cell variability and drug-induced reprogramming as a mode of cancer drug resistance. Nature. Nature Publishing Group; 2017;546:431-5.

23. Roesch A, Vultur A, Bogeski I, Wang H, Zimmermann KM, Speicher D, et al. Overcoming Intrinsic Multidrug Resistance in Melanoma by Blocking the Mitochondrial Respiratory Chain of Slow-Cycling JARID1Bhigh Cells. Cancer Cell. 2013;23:811-25.

24. Rambow F, Rogiers A, Marin-Bejar O, Aibar S, Femel J, Dewaele M, et al. Toward Minimal Residual Disease-Directed Therapy in Melanoma. Cell. Elsevier Inc.; 2018;174:843855.e19.

25. Ku SY, Rosario S, Wang Y, Mu P, Seshadri M, Goodrich ZW, et al. Rb1 and Trp53 cooperate to suppress prostate cancer lineage plasticity, metastasis, and antiandrogen resistance. Science (80- ). 2017;355:78-83.

26. $\mathrm{Mu}$ P, Zhang Z, Benelli M, Karthaus WR, Hoover E, Chen C-C, et al. SOX2 promotes lineage plasticity and antiandrogen resistance in TP53- and RB1-deficient prostate cancer. Science (80- ). 2017;355:84-8.

27. Burris H a, Hurwitz HI, Dees EC, Dowlati A, Blackwell KL, O’Neil B, et al. Phase I safety, pharmacokinetics, and clinical activity study of lapatinib (GW572016), a reversible dual inhibitor of epidermal growth factor receptor tyrosine kinases, in heavily pretreated patients with metastatic carcinomas. J Clin Oncol. 2005;23:5305-13.

28. O'Brien N a, Browne BC, Chow L, Wang Y, Ginther C, Arboleda J, et al. Activated phosphoinositide 3-kinase/AKT signaling confers resistance to trastuzumab but not lapatinib. Mol Cancer Ther. 2010;9:1489-502.

29. DepMap B. DepMap 20Q1 Public. 2020.

30. Gymnopoulos M, Elsliger M-A, Vogt PK. Rare cancer-specific mutations in PIK3CA show gain of function. Proc Natl Acad Sci U S A. 2007;104:5569-74.

31. Ikenoue T, Kanai F, Hikiba Y, Obata T, Tanaka Y, Imamura J, et al. Functional analysis of PIK3CA gene mutations in human colorectal cancer. Cancer Res. 2005;65:4562-7.

32. Bhang HC, Ruddy D a, Krishnamurthy Radhakrishna V, Caushi JX, Zhao R, Hims MM, et al. Studying clonal dynamics in response to cancer therapy using high-complexity barcoding. Nat Med. 2015; 
33. Khodadadi-Jamayran A, Pucella J, Zhou H, Doudican N, Carucci J, Heguy A, et al. iCellR: Combined Coverage Correction and Principal Component Alignment for Batch Alignment in Single-Cell Sequencing Analysis. bioRxiv. 2020;2020.03.31.019109.

34. Xue JY, Zhao Y, Aronowitz J, Mai TT, Vides A, Qeriqi B, et al. Rapid non-uniform adaptation to conformation-specific $\operatorname{KRAS}(\mathrm{G} 12 \mathrm{C})$ inhibition. Nature. Springer US; 2020;577:421-5.

35. Spencer SL, Cappell SD, Tsai F-C, Overton KW, Wang CL, Meyer T. The proliferationquiescence decision is controlled by a bifurcation in CDK2 activity at mitotic exit. Cell. Elsevier Inc.; 2013;155:369-83.

36. Mora A, Komander D, van Aalten DMF, Alessi DR. PDK1, the master regulator of AGC kinase signal transduction. Semin Cell Dev Biol. 2004;15:161-70.

37. Wang Y, Zhou D, Phung S, Masri S, Smith D, Chen S. SGK3 is an estrogen-inducible kinase promoting estrogen-mediated survival of breast cancer cells. Mol Endocrinol. 2011;25:72-82.

38. Bago R, Sommer E, Castel P, Crafter C, Bailey FP, Shpiro N, et al. The hVps34-SGK3 pathway alleviates sustained PI3K/Akt inhibition by stimulating mTORC1 and tumour growth. EMBO J. 2016;35:1902-22.

39. Inoki K, Li Y, Zhu T, Wu J, Guan K-L. TSC2 is phosphorylated and inhibited by Akt and suppresses mTOR signalling. Nat Cell Biol. 2002;4:648-57.

40. Manning BD, Tee AR, Logsdon MN, Blenis J, Cantley LC. Identification of the tuberous sclerosis complex-2 tumor suppressor gene product tuberin as a target of the phosphoinositide 3-kinase/akt pathway. Mol Cell. 2002;10:151-62.

41. Menon S, Dibble CC, Talbott G, Hoxhaj G, Valvezan AJ, Takahashi H, et al. Spatial control of the TSC complex integrates insulin and nutrient regulation of mtorc 1 at the lysosome. Cell. Elsevier Inc.; 2014;156:1771-85.

42. Castel P, Ellis H, Bago R, Toska E, Razavi P, Carmona FJ, et al. PDK1-SGK1 Signaling Sustains AKT-Independent mTORC1 Activation and Confers Resistance to PI3K $\alpha$ Inhibition. Cancer Cell. 2016;1-14.

43. Zhang HH, Huang J, Düvel K, Boback B, Wu S, Squillance RM, et al. Insulin stimulates adipogenesis through the Akt-TSC2-mTORC1 pathway. PLoS One. 2009;4.

44. Halland N, Schmidt F, Weiss T, Saas J, Li Z, Czech J, et al. Discovery of N-[4-(1Hpyrazolo[3,4-b]pyrazin-6-yl)-phenyl]-sulfonamides as highly active and selective SGK1 inhibitors. ACS Med Chem Lett. 2015;6:73-8.

45. Murray JT, Campbell DG, Morrice N, Auld GC, Shpiro N, Marquez R, et al. Exploitation of KESTREL to identify NDRG family members as physiological substrates for SGK1 and GSK3. Biochem J. 2004;384:477-88.

46. Hata AN, Niederst MJ, Archibald HL, Gomez-Caraballo M, Siddiqui FM, Mulvey HE, et al. Tumor cells can follow distinct evolutionary paths to become resistant to epidermal growth factor receptor inhibition. Nat Med. 2016;22.

47. Ramirez M, Rajaram S, Steininger RJ, Osipchuk D, Roth MA, Morinishi LS, et al. Diverse drug-resistance mechanisms can emerge from drug-tolerant cancer persister cells. Nat Commun. Nature Publishing Group; 2016;7:10690.

48. Harbeck N, Gluz O, Christgen M, Kates RE, Braun M, Küemmel S, et al. De-escalation strategies in human epidermal growth factor receptor 2 (HER2)-positive early breast cancer (BC): Final analysis of the West German study group Adjuvant Dynamic Marker-Adjusted Personalized Therapy Trial Optimizing Risk Assessment and Therapy. J Clin Oncol. 
2017;35:3046-54.

49. Berns K, Horlings HM, Hennessy BT, Madiredjo M, Hijmans EM, Beelen K, et al. A functional genetic approach identifies the PI3K pathway as a major determinant of trastuzumab resistance in breast cancer. Cancer Cell. 2007;12:395-402.

50. Nagata Y, Lan K-H, Zhou X, Tan M, Esteva FJ, Sahin A a, et al. PTEN activation contributes to tumor inhibition by trastuzumab, and loss of PTEN predicts trastuzumab resistance in patients. Cancer Cell. 2004;6:117-27.

51. The Cancer Genome Atlas. Comprehensive molecular portraits of human breast tumours. Nature. 2012;490:61-70.

52. Watson SS, Dane M, Chin K, Tatarova Z, Liu M, Liby T, et al. Microenvironment-Mediated Mechanisms of Resistance to HER2 Inhibitors Differ between HER2+ Breast Cancer Subtypes. Cell Syst. Elsevier Inc.; 2018;6:329-342.e6.

53. National Comprehensive Caner Network. NCCN clinical practice guidelines in oncology: breast cancer Version 2.2019 [Internet]. Available from: https://www.nccn.org/professionals/physician_gls/pdf/breast.pdf

54. Recasens A, Munoz L. Targeting Cancer Cell Dormancy. Trends Pharmacol Sci. Elsevier Ltd; 2019;40:128-41.

55. Vallette FM, Olivier C, Lézot F, Oliver L, Cochonneau D, Lalier L, et al. Dormant, quiescent, tolerant and persister cells: Four synonyms for the same target in cancer. Biochem Pharmacol. Elsevier; 2019;162:169-76.

56. Aguirre-Ghiso JA, Sosa MS. Emerging Topics on Disseminated Cancer Cell Dormancy and the Paradigm of Metastasis. Annu Rev Cancer Biol. 2018;2:377-93.

57. Ghajar CM. Metastasis prevention by targeting the dormant niche. Nat Rev Cancer. 2015;15:238-47.

58. Reya T, Morrison SJ, Clarke MF, Weissman IL. Stem cells, cancer, and cancer stem cells. Nature. 2001;414:105-11.

59. Wang JCY, Dick JE. Cancer stem cells: Lessons from leukemia. Trends Cell Biol. 2005;15:494-501.

60. Chaffer CL, Marjanovic ND, Lee T, Bell G, Kleer CG, Reinhardt F, et al. Poised chromatin at the ZEB1 promoter enables breast cancer cell plasticity and enhances tumorigenicity. Cell. Elsevier Inc.; 2013;154:61-74.

61. Chaffer CL, Brueckmann I, Scheel C, Kaestli AJ, Wiggins P a, Rodrigues LO, et al. Normal and neoplastic nonstem cells can spontaneously convert to a stem-like state. Proc Natl Acad Sci U S A. 2011;108:7950-5.

62. Roesch A, Fukunaga-Kalabis M, Schmidt EC, Zabierowski SE, Brafford P a, Vultur A, et al. A temporarily distinct subpopulation of slow-cycling melanoma cells is required for continuous tumor growth. Cell. Elsevier Ltd; 2010;141:583-94.

63. Moser J, Miller I, Carter D, Spencer SL. Control of the Restriction Point by Rb and p21. Proc Natl Acad Sci. 2018;115:E8219-27.

64. Tirosh I, Izar B, Prakadan SM, Wadsworth MH, Treacy D, Trombetta JJ, et al. Dissecting the multicellular ecosystem of metastatic melanoma by single-cell RNA-seq. Science (80- ). 2016;352:189-96.

65. Travnickova J, Wojciechowska S, Khamseh A, Gautier P, Brown D V., Lefevre T, et al. Zebrafish MITF-low melanoma subtype models reveal transcriptional subclusters and MITF-independent residual disease. Cancer Res. 2019;79:5769-84.

66. Crea F, Nur Saidy NR, Collins CC, Wang Y. The epigenetic/noncoding origin of tumor 
dormancy. Trends Mol Med. Elsevier Ltd; 2015;21:206-11.

67. Oki T, Nishimura K, Kitaura J, Togami K, Maehara A, Izawa K, et al. A novel cell-cycleindicator, mVenus-p27K -, identifies quiescent cells and visualizes G0-G1 transition. Sci Rep. 2014;4.

68. Becker W. A wake-up call to quiescent cancer cells - potential use of DYRK1B inhibitors in cancer therapy. FEBS J. 2018;285:1203-11.

69. Marcotte R, Sayad A, Brown KR, Sanchez-Garcia F, Reimand J, Haider M, et al. Functional Genomic Landscape of Human Breast Cancer Drivers, Vulnerabilities, and Resistance. Cell. 2016;164:293-309.

70. Hirai H, Sootome H, Nakatsuru Y, Miyama K, Taguchi S, Tsujioka K, et al. MK-2206, an allosteric Akt inhibitor, enhances antitumor efficacy by standard chemotherapeutic agents or molecular targeted drugs in vitro and in vivo. Mol Cancer Ther. 2010;9:1956-67.

71. Loibl S, de la Pena L, Nekljudova V, Zardavas D, Michiels S, Denkert C, et al. Neoadjuvant buparlisib plus trastuzumab and paclitaxel for women with HER2+ primary breast cancer: A randomised, double-blind, placebo-controlled phase II trial (NeoPHOEBE). Eur J Cancer. 2017;85:133-45.

72. Wang Y, Zhou D, Phung S, Warden C, Rashid R, Chan N, et al. SGK3 sustains ER $\alpha$ signaling and drives acquired aromatase inhibitor resistance through maintaining endoplasmic reticulum homeostasis. Proc Natl Acad Sci. 2017;201612991.

73. Xu J, Wan M, He Q, Bassett RL, Fu X, Chen AC, et al. SGK3 is associated with estrogen receptor expression in breast cancer. Breast Cancer Res Treat. 2012;134:531-41.

74. Sommer EM, Dry H, Cross D, Guichard S, Davies BR, Alessi DR. Elevated SGK1 predicts resistance of breast cancer cells to Akt inhibitors. Biochem J. 2013;452:499-508.

75. Bago R, Malik N, Munson MJ, Prescott AR, Davies P, Sommer E, et al. Characterization of VPS34-IN1, a selective inhibitor of Vps34, reveals that the phosphatidylinositol 3phosphate-binding SGK3 protein kinase is a downstream target of class III phosphoinositide 3-kinase. Biochem J. 2014;427:413-27.

76. Wu X, Simpson J, Hong JH, Kim K, Thavarajah NK, Backx PH, et al. MEK-ERK pathway modulation ameliorates disease phenotypes in a mouse model of Noonan syndrome associated with the Raf1L613V mutation. J Clin Invest. 2011;121:1009-25.

77. McInnes L, Healy J, Saul N, Großberger L. UMAP: Uniform Manifold Approximation and Projection. J Open Source Softw. 2018;3:861.

78. Bern YK Becker, C MK. Byonic: advanced peptide and protein identification software, Curr Protoc Bioinformatics. Curr Protoc Bioinforma Chapter 13. 2012; Unit13.

79. Bern M, Cai Y, Goldberg D. Lookup peaks: a hybrid of de novo sequencing and database search for protein identification by tandem mass spectrometry. Anal Chem. 2007;79:1393400 . 


\section{Figure Legends}

Figure 1. Some HER2+ breast cancer cell lines give rise to lapatinib drug-tolerant persisters (lapatinib-DTPs). A, HER2+ breast cancer cell lines were treated with $2.5 \mu \mathrm{M}$ lapatinib, counted on a Vi-Cell counter at the indicated times, and normalized to the initial cell number. Means \pm SEM from three independent experiments are shown. The inset shows a magnified view of the residual cells from Days 28-49. B, HER2+ breast cancer lines were cultured in lapatinib for 14 days to generate DTPs. Then, lapatinib was withdrawn and cells were allowed to proliferate drugfree. At the indicated times, cells were re-challenged with $2.5 \mu \mathrm{M}$ lapatinib. Parental cells were used as a control at each time point. Cell survival were assessed by counting viable cells after 7day lapatinib treatment and normalized to the viable cell number at Day $0(t=0)$. Means \pm SEM from three independent experiments are displayed. Statistical significance was assessed by twotailed t-test for SUM-225. All other cell lines were assessed by two-way ANOVA with Bonferroni post-hoc analysis $\left({ }^{*} p>0.05, * * p<0.01, * * * p<0.001, * * * * p<0.0001\right)$. C, Number of tumors detected and estimated tumor-initiating cell (TIC) frequency of BT474 parental and DTPs after injection into the mammary fat pad of NSG mice. The image at the right shows tumors that arose by 5 months after injection.

Figure 2. HER2+ breast cancer cells elicit distinct transcriptional programs after lapatinib treatment. A, Heat map shows unsupervised clustering of parental and DTP samples. Counts from technical duplicates were merged in this analysis. Scale represents the z-score. $\mathbf{B}$ and $\mathbf{C}$, Top and bottom 10 enriched GSEA terms for differentially expressed genes (DEGs) between DTP and parental cells in the mesenchymal-like (B) and luminal-like (C) subgroups. Enriched terms are ranked by Normalized Enrichment Score (NES). D, Alamar Blue readings of SKBR3, EFM192A, BT474, and HCC1419 cells treated as indicated for 7 days. Relative fluorescence units (RFU) were normalized to $\mathrm{t}=0$. Means $\pm \mathrm{SEM}$ of three independent experiments are displayed. $* * * * \mathrm{p}<0.0001$, two-tailed t-test. E, Change in DTP numbers following fulvestrant plus lapatinib treatment compared to lapatinib treatment alone. Means + SEM of three independent experiments are displayed. Statistical significance was assessed by two-tailed t-test $(* * * * p<0.0001) \mathbf{F}$, Incucyte images of SKBR3, EFM192A, BT474, and HCC1419 cells treated as indicated for 14 days and then cultured drug-free for 14 days to assess regrowth. Representative images from three independent experiments are displayed. G, The DESeq2 normalized expression of ESR1 is displayed for parental cells and DTPs of each cell line.

Figure 3. A fraction of randomly growing HER2-luminal cells occupies a pre-DTP state characterized by a $\mathbf{G}_{0}$-like signature. A, Aggregate expression of BT474-DTP Up and Down DEG in single cells from untreated (UT), 6-hour lapatinib-treated (6h) and DTP (14-day lapatinib) samples. B, UMAP projection of unsupervised hierarchical clustering of single cell gene expression in untreated BT474 cells. Cells are colored by their respective cluster. C, Aggregate expression of BT474-DTP Up and Down DEGs, as indicated, in each unsupervised cluster of untreated BT474 cells from B. D, UMAP projection of supervised clustering of untreated BT474 cells using cell cycle signature genes as defined by Xue et al. Cells are colored by cell cycle stage (see Methods for details). E, UMAP projection showing supervised clustering of untreated BT474 cells with Xue et al. cell cycle genes (as in D), but with cells colored according to their unsupervised clusters (as determined in B). F, Same projection as in D, but with pre-DTPs (based on their BT474 combined score) shown as larger circles compared with other cells. G, Heat map 
displaying BT474 Up DEG score, BT474 Down DEG score and BT474 combined DEG score (up DEG score minus down DEG score), in single untreated BT474 cells. The upper panels show the unsupervised clusters from B and their cell cycle phase, determined by expression of the Xue et al. cell cycle genes. H, UMAP projection of supervised clustering of untreated HCC1419 cells by their expression of cell cycle genes. Cells are colored by cell cycle status. I, Heat map displaying HCC1419 Up DEG score, HCC1419 Down DEG score, and HCC1419 combined DEG score (up minus down DEG score) in single HCC1419 cells. Upper panels display the unsupervised cluster to which each untreated HCC1419 cell belongs and its cell cycle phase.

Figure 4. NPY1R enables prospective purification of pre-DTPs A, Overlap of untreated BT474 cluster B DEGs with fold change (FC) $>1.5$ and BT474-DTP DEGs with FC $>2$. The 20 overlapping genes and their respective p-values (Fisher's exact test) are displayed. Genes encoding surface proteins are shown in bold. B, FACS-isolated NPY1R ${ }^{\text {hi }}$ NPY1R ${ }^{\text {mid }}$ and NPY1R ${ }^{\text {lo }}$ cells were treated with lapatinib for 14 days. Average RFU indicates the Alamar Blue readings at the experimental endpoint. Means_SEMs for three independent experiments are shown. Significance was evaluated by two-tailed t-test $(* * p<0.01, * * * * p<0.0001)$. C, Overlap of NPY1R and BT474DTP DEGs with directionality of the DEGs matched in the analysis and p-value (Fisher's exact test) displayed. D, GSEA showing enrichment for "Hallmark Estrogen Response Early" genes in NPY1R ${ }^{\text {hi }}$ versus NPY1R ${ }^{\text {lo }}$ cells. E, qRT-PCR for NPY1R in FACS-isolated NPY1R ${ }^{\text {hi }}$, NPY1R ${ }^{\text {mid }}$ and NPY $1 R^{\text {lo }}$ cells that were either left untreated, treated with lapatinib for 6 hours (Lap), or treated with lapatinib and fulvestrant for 6 hours (Lap + Ful). Relative NPY1R expression represents the $2^{-} \triangle \Delta \mathrm{CT}$ relative to the untreated NPY1R ${ }^{\text {lo }}$ sample. The means $\pm \mathrm{SEM}$ of three independent experiments are displayed (ns, $\mathrm{p}>0.05,{ }^{*} \mathrm{p}<0.01, * * * * \mathrm{p}<0.0001$, two-tailed t-test). F, BT474 cells were stained with Hoechst 33342 and the cells from NPY1R ${ }^{\text {hi }}$ and NPY $1 R^{\text {lo }}$ fractions are displayed. G, Quantification of cells in $\mathrm{G}_{0} / \mathrm{G} 1$ and $\mathrm{S}-\mathrm{G} 2 \mathrm{M}$ from panel F. (H and I) Pseudotime analysis of cells from untreated BT474 cells, 6h lap-treated cells and DTP samples with cells colored by sample origin (H) or NPY1R expression (I). J, Cells from BT474 untreated and 6h lap sample were isolated (computationally), and their respective positions on the pseudotime trajectory are displayed. Cells are colored by their relative NPY1R expression level. $\mathbf{K}$, Cells from untreated and 6 lapatinib-treated BT474 cells were isolated (computationally), and their respective positions on the pseudotime trajectory are displayed. Cells are colored by their cell cycle phase.

Figure 5. Lapatinib-DTPs are more sensitive to PI3K than AKT inhibition. (A and B) BT474DTPs (A) and HCC1419-DTPs (B) were treated with increasing doses of BKM120 (pan-PI3K inhibitor), GSK690693 (AKT kinase inhibitor) and MK-2206 (allosteric inhibitor of AKT) for 96 $\mathrm{hr}$, and cell number was determined by Alamar Blue viability assay. Means $\_$SEM from three independent experiments are shown. (C and D) Differential effect of PI3K and AKT inhibitors on PI3K pathway components. Cells were treated with the indicated inhibitors or vehicle (DMSO) for one hour before lysis. Whole cell lysates were resolved by SDS-PAGE and immunoblotted with the indicated antibodies to assess pathway activation. Numbers under blots represent relative band intensities compared to vehicle control. Representative blots from two independent experiments are shown.

Figure 6. Luminal-like lapatinib-DTPs up-regulate $S G K 3$ via estrogen receptor action. A, $S G K 3$ expression was quantified by qRT-PCR of mRNA from the indicated parental cells and DTPs. Data represent means + SEM from three independent experiments $(* p<0.05, * * * p<0.001$, 
$* * * * p<0.0001$, two-tailed t test). B, SGK3 levels in whole cell lysates from the indicated parental cells and DTPs, quantified by immunoblotting. ERK2 serves as a loading control. Numbers under blots represent the normalized relative intensity compared to parental cells. Representative blots from three independent experiments are shown. (C and D) $S G K 3$ expression, quantified by qRTPCR, from BT474 (C) and HCC1419 (D) cells treated with DMSO vehicle (veh), lapatinib (Lap), fulvestrant (Ful), or lapatinib plus fulvestrant (Lap + Ful) for 48 hours. Means and SEM \pm three independent experiments are displayed. $(* \mathrm{p}<0.05, * * \mathrm{p}<0.01$, two-tailed t-test). (E and $\mathbf{F})$ Immunoblots of whole cell lysates from BT474 (E) and HCC1419 (F) cells, treated as indicated for 48 hours. Representative blots from two independent experiments are displayed.

Figure 7. SGK3 phosphorylates TSC2 to activate mTORC1. A, Flag-tagged TSC2 was transfected into $293 \mathrm{~T}$ cells, which were then treated with $1 \mu \mathrm{M}$ BKM120 for 1 hour to allow dephosphorylate PI3K-dependent sites. Cells were lysed and TSC2 was recovered by immunoprecipitation with ANTI-FLAG M2 agarose beads and incubated with $100 \mathrm{ng}$ recombinant $\mathrm{SGK} 3$ at $30^{\circ} \mathrm{C}$ for 30 minutes in presence of $100 \mu \mathrm{M}$ ATP. Immunoprecipitates and lysates were subjected to immunoblot analysis with the indicated antibodies. Representative blots from two independent experiments are shown. B, A scaled-up in vitro kinase reaction was performed as described in A and analyzed buy mass spectrometry. Results show relative phosphorylation for each pAKT motif site, compared with the TSC2-only control. C, Schematic showing wild type (wt) TSC2 and positions of S/T->A TSC2 phosphorylation mutants. Sites conforming to the AKT substrate motif sequence (RXRXXS/T) are displayed with reported AKT phosphorylation sites underlined. SGK3 sites identified by mass spectrometry are highlighted in red. Specific S/T- $>$ A mutants analyzed also are shown. D, 293T cells were transfected with expression constructs for flag-tagged wild type or mutant TSC2 with or without SGK3-GST, as indicated, and then serumstarved overnight. Where indicated, cells were treated with $1 \mu \mathrm{M}$ MK-2206 for 30 minutes prior to stimulation with $50 \mathrm{ng} / \mathrm{ml} \mathrm{IGF1}$ for 30 minutes. Cells were then lysed, and whole cell lysates and TSC2 immunoprecipitates were immunoblotted with the indicated antibodies. Numbers under the blots represent relative intensities compared to that from cells co-transfected with wt-TSC2 and SGK3-GST + MK-2206; signals from the wt-TSC2 + MK-2206 lanes were subtracted from each before quantification. Representative blots from one of two independent experiments are displayed. (E and G) BT474-DTPs (E) and HCC1419-DTPs (G) were treated alone or in combination with $3 \mu \mathrm{M}$ or $10 \mu \mathrm{M}$ BKM120, $1 \mu \mathrm{M}$ GSK690693, $1 \mu \mathrm{M}$ MK-2206, or $3 \mu \mathrm{M}$ 14h for 96h, and cell survival was assessed by Alamar Blue assay. Means \pm SEM of the normalized relative fluorescence units from three independent experiments are displayed $\left({ }^{*} \mathrm{p}<0.05,{ }^{*} \mathrm{p}<0.01\right.$, two-tailed t test). (F and H) BT474-DTPs (F) and HCC1419-DTPs (H) were treated with the inhibitors for one hour, and whole cell lysates were subjected to immunoblot analysis with the indicated antibodies. Numbers under the blots indicate relative intensity compared with the vehicle control. Representative blots from two independent experiments are displayed. 
A
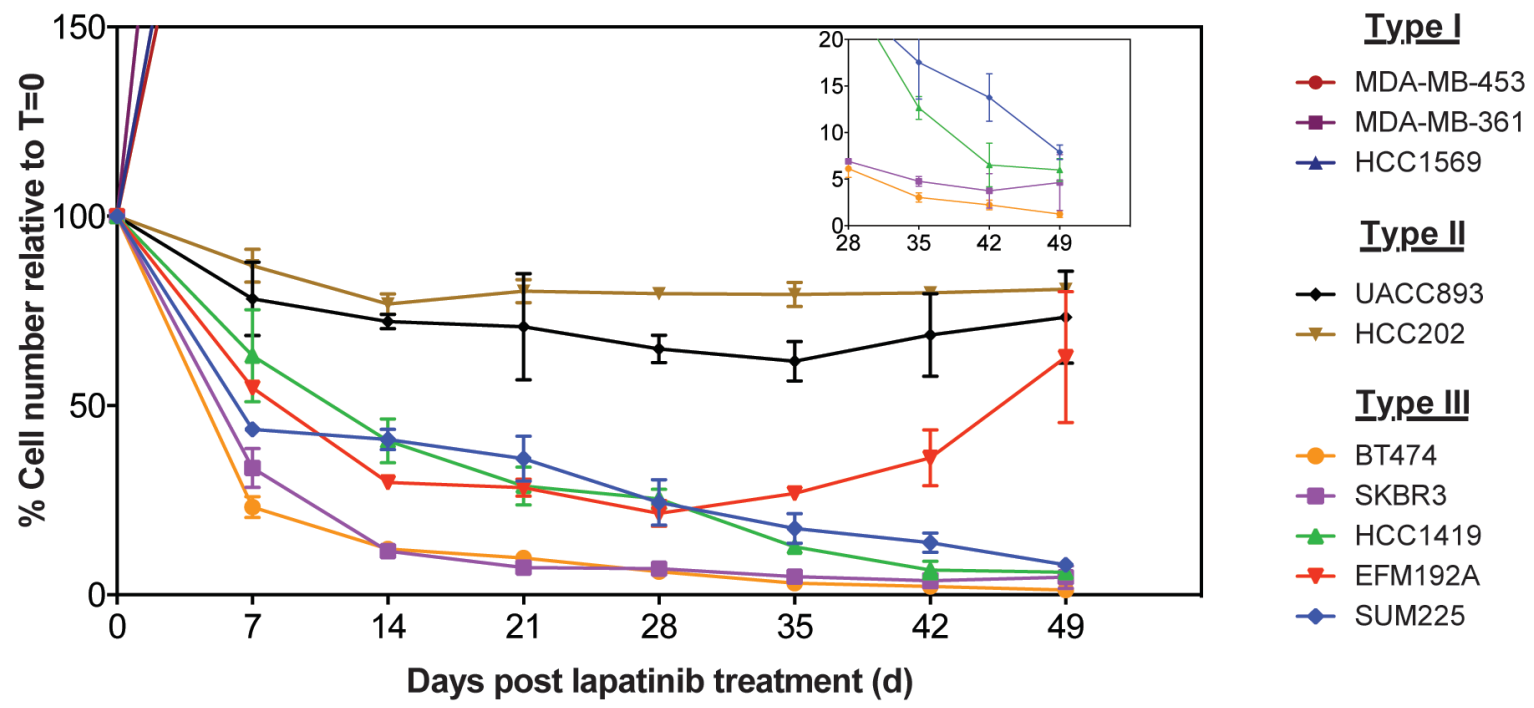

B

BT474
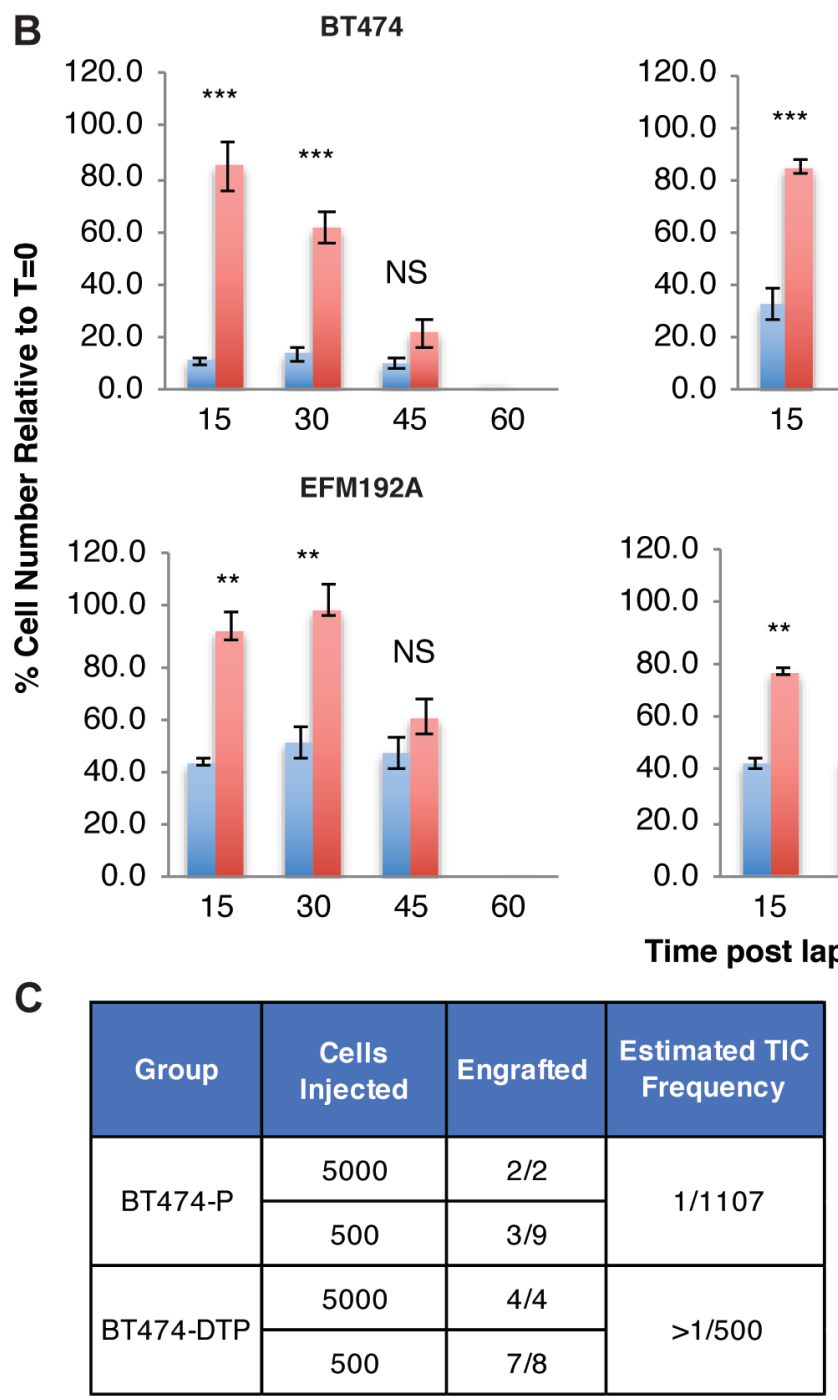

SKBR3

SUM225

C

\begin{tabular}{|c|c|c|c|}
\hline Group & $\begin{array}{c}\text { Cells } \\
\text { Injected }\end{array}$ & Engrafted & $\begin{array}{c}\text { Estimated TIC } \\
\text { Frequency }\end{array}$ \\
\hline \multirow{2}{*}{ BT474-P } & 5000 & $2 / 2$ & \multirow{2}{*}{$1 / 1107$} \\
\cline { 2 - 3 } & 500 & $3 / 9$ & \\
\hline \multirow{2}{*}{ BT474-DTP } & 5000 & $4 / 4$ & \multirow{2}{*}{$>1 / 500$} \\
\cline { 2 - 3 } & 500 & $7 / 8$ & \multicolumn{2}{|c|}{} \\
\hline
\end{tabular}

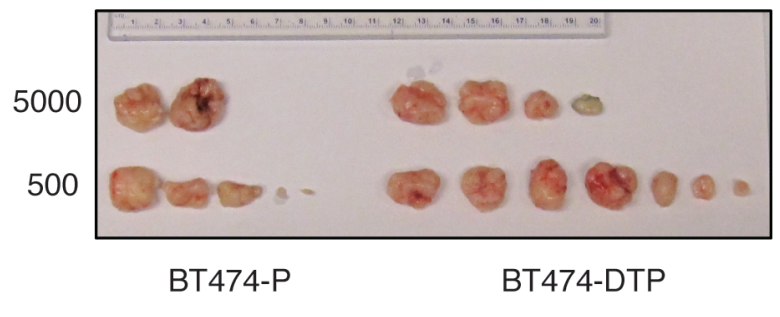


Figure 2

A

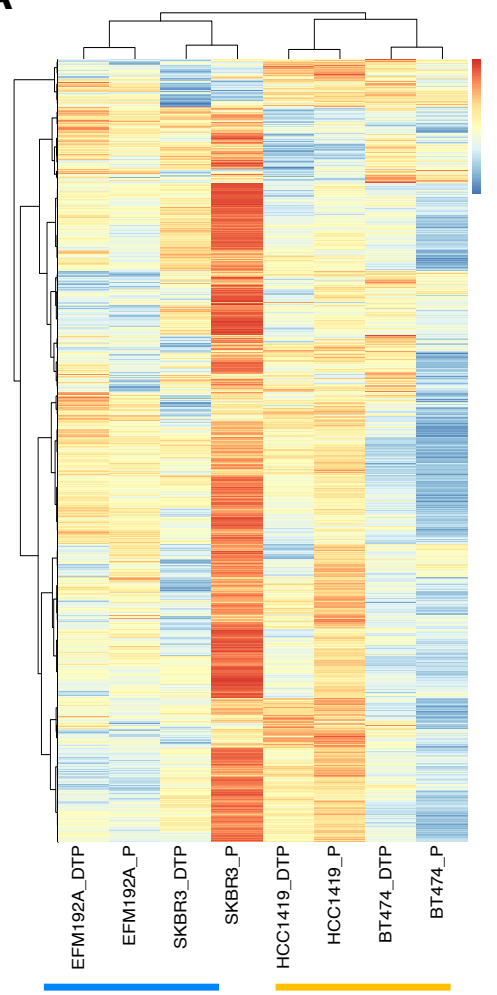

B
Mesenchymal-like DTP GSEA NES

Extracellular Matrix Organization

Extracellular Structure Organization

Naba Core Matrisome

Extracellular Matrix Organization

Hallmark Coagulation

Integrin Cell Surface Interactions

Naba ECM Glycoproteins

Degradation Of The Extracellular
Matrix

Peptide Cross-linking

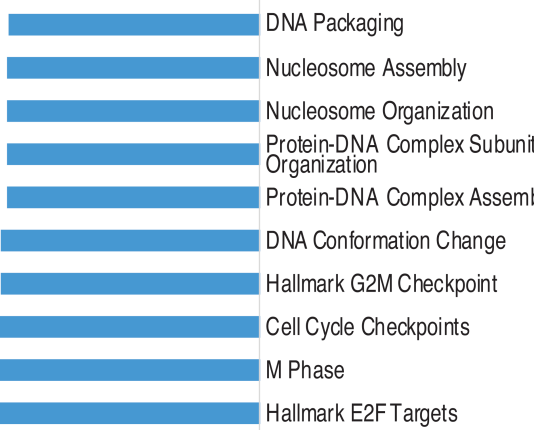
$-2$
0
2

Hallmark Epithelial Mesenchymal

C

$\begin{array}{llllllllll}-2.5 & -2 & -1.5 & -1 & -0.5 & 0 & 0.5 & 1 & 1.5 & 2\end{array}$

Hallmark Estrogen Response Early

Detection Of Stimulus Involved In Sensory Perception

Regulation Of Blood Coagulation

Regulation Of Hemostasis

Regulation Of Coagulation

Detection Of Chemical Stimulus Involved

In Sensory Perception

Defective B3galt I Causes Peters-plus

Response To Exogenous DsRNA

Positive Regulation Of Lipid Kinase

$$
\text { Activity }
$$

Regulation Of Response To Wounding
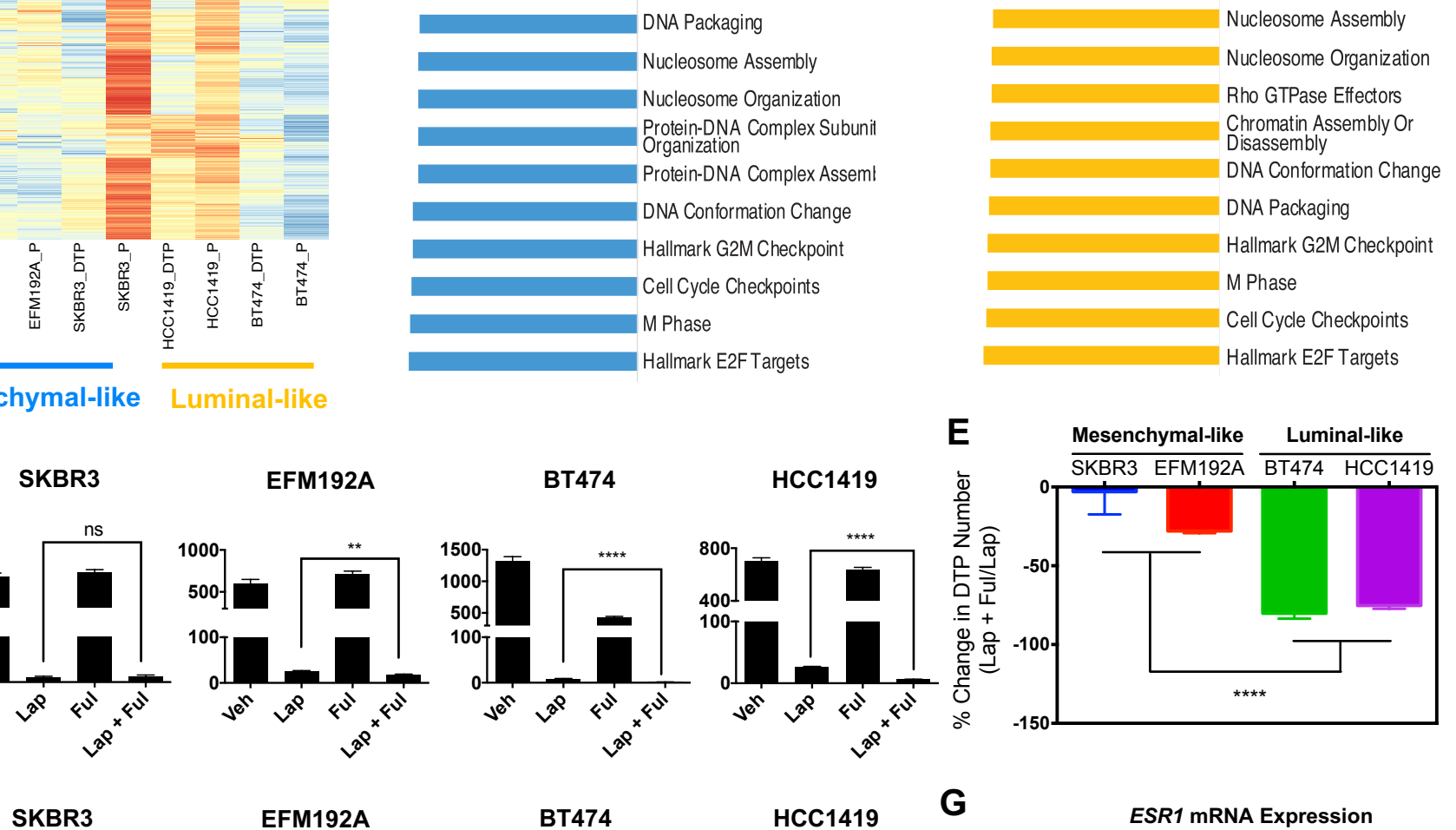

EFM192A

BT474

HCC1419

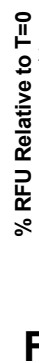

SKBR3

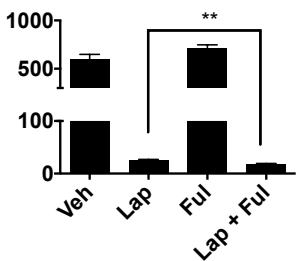

EFM192A

Veh Lap

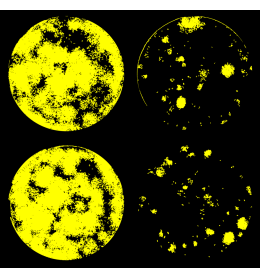

Ful Ful+Lap

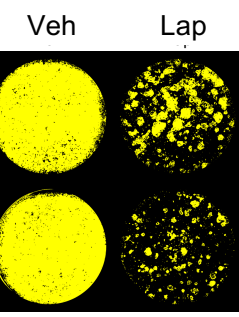

Ful Ful+Lap

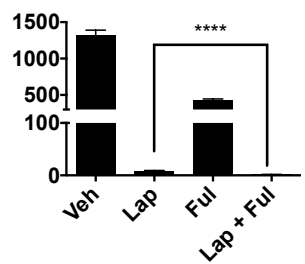

BT474

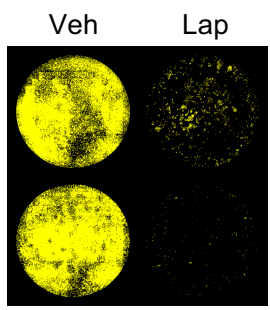

Fül Fül+Lap

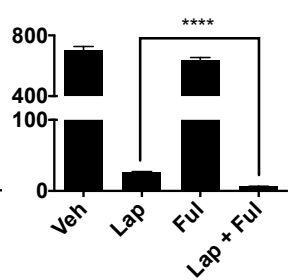

G

HCC1419

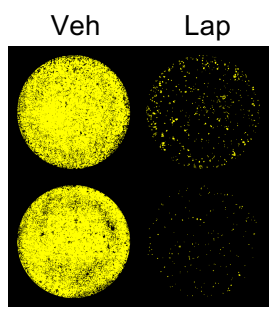

Fü Fül+Lap
ESR1 mRNA Expression

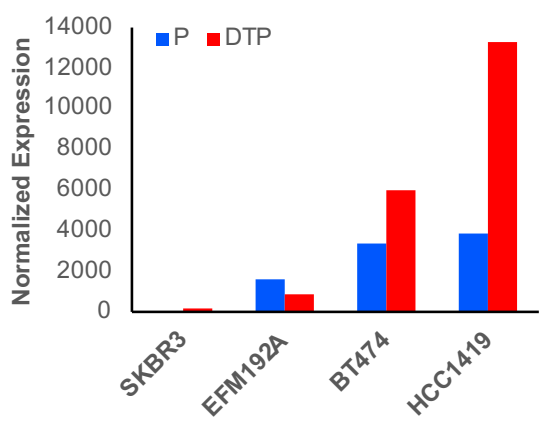




\section{Figure 3}

A

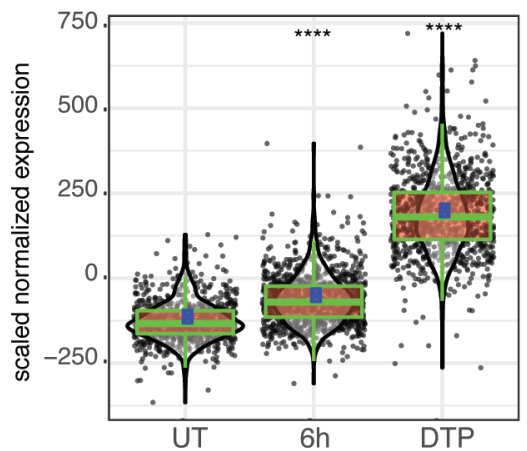

C

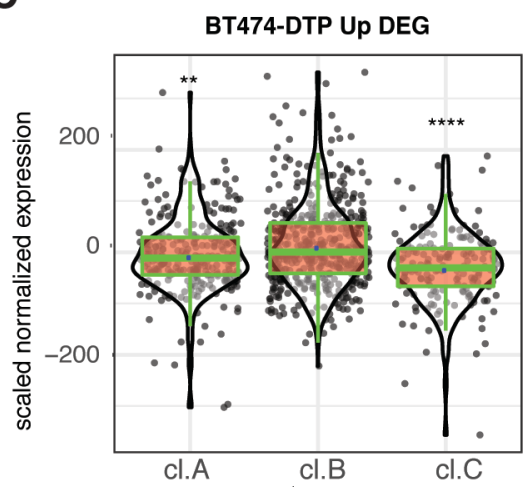

$\mathbf{F}$
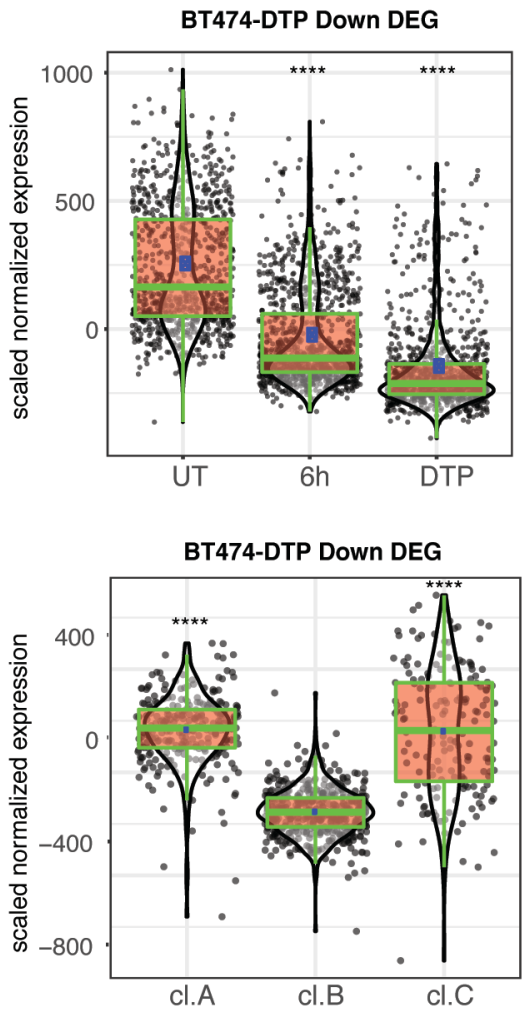

G
B

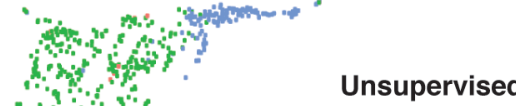
Clusters

- $\mathrm{A}$

- B
D

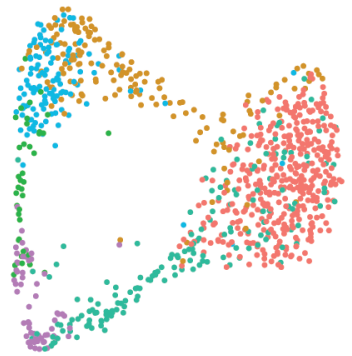

E

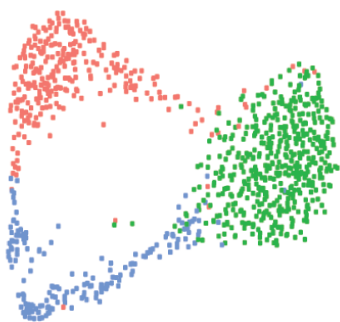

Cell Cycle

Phases

- G0

- G1S

- G2M

- M

- MG1

- $\mathrm{S}$
Unsupervised Clusters

A

$\because \mathrm{B}$
Cell Cycle

Phases

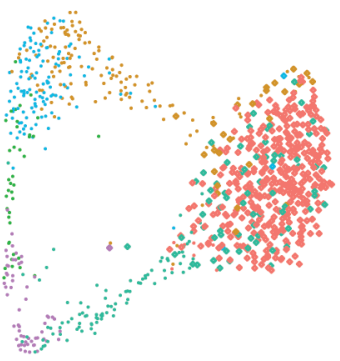

- G0

- G1S

- G2M

- $M$

- MG

- $S$

Pre-DTP

- pre-DTP rest

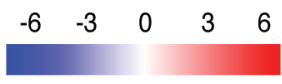

Signature Score

Unsupervised_clusters Cell_Cycle_Phases

BT_up_score

BT_down_score

BT_combined_score

HCC1419 UT

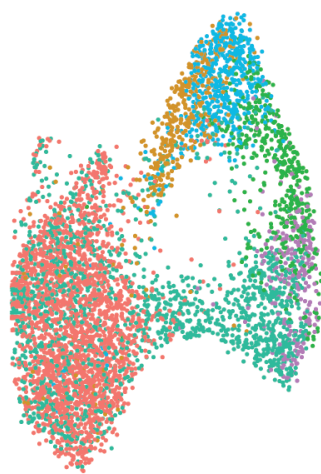

Cell Cycle Phases

- G0

- G1S

- G2M

- M

- MG1

- $\mathrm{S}$

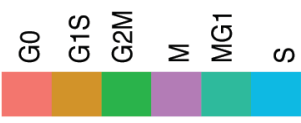

Cell Cycle Phases
Unsupervised_clusters Cell_Cycle_Phases

HCC_up_score HCC_down_score HCC_combined_score

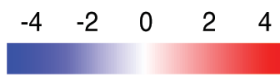

Signature Score

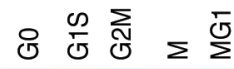

Cell Cycle Phases 
Figure 4

A

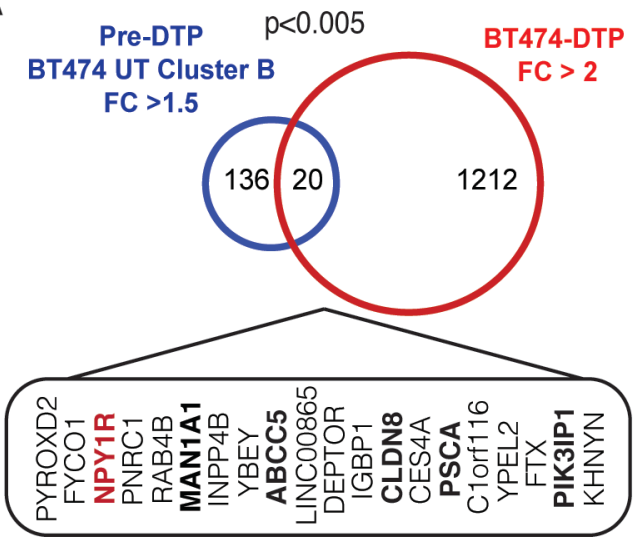

B

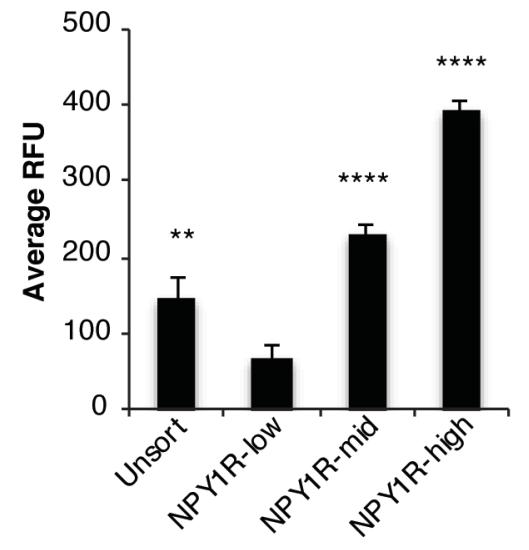

E

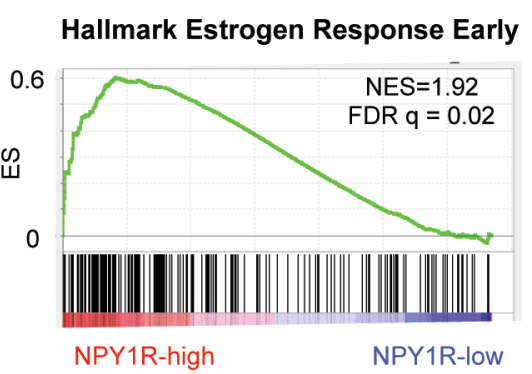

$\mathbf{F}$
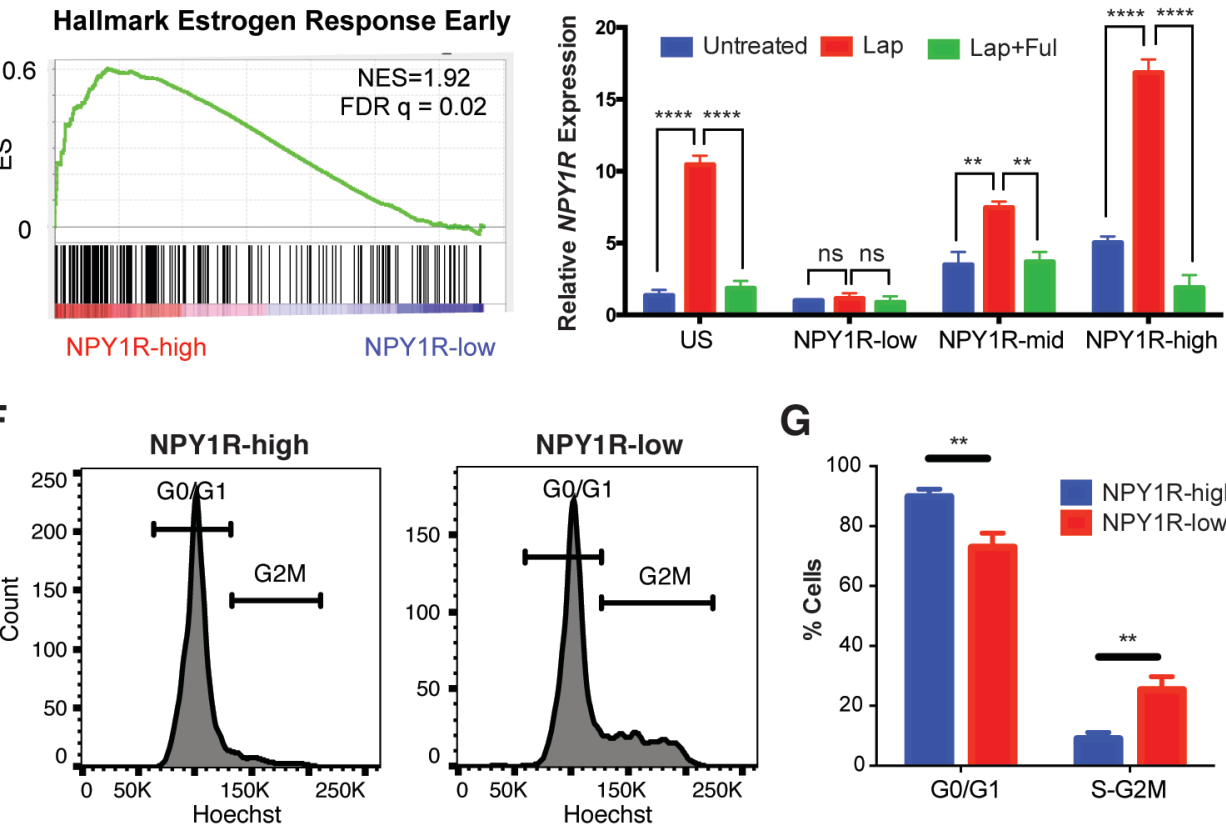

J

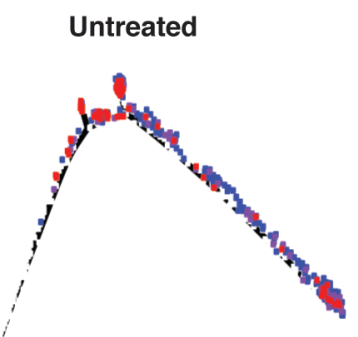

DTP2

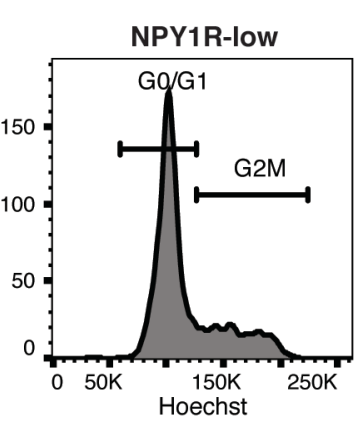

G

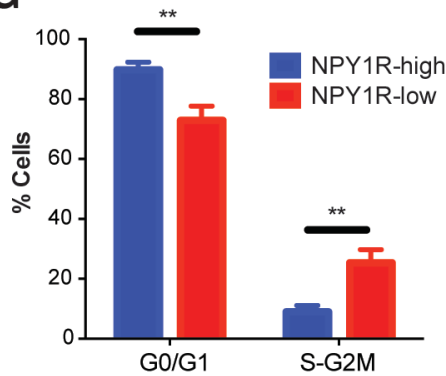

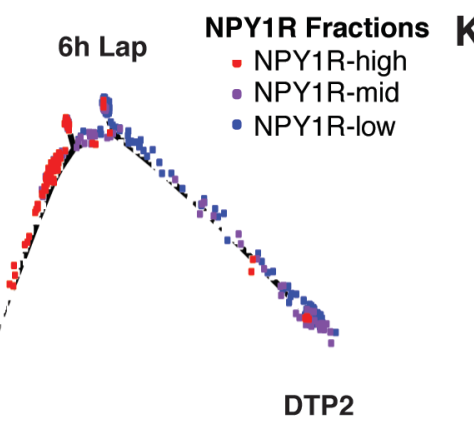

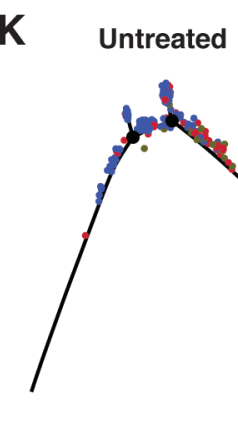

C

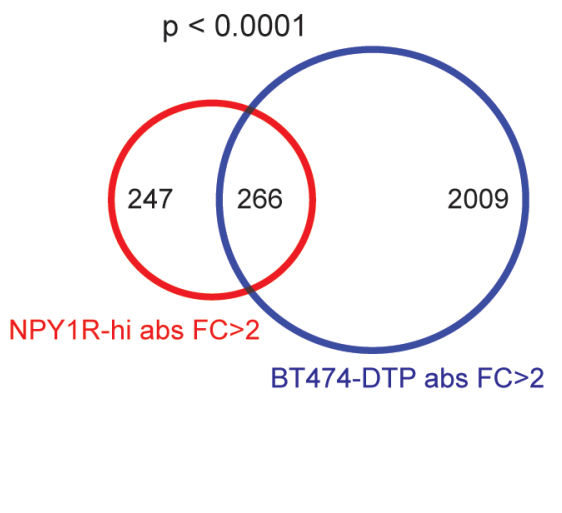

H

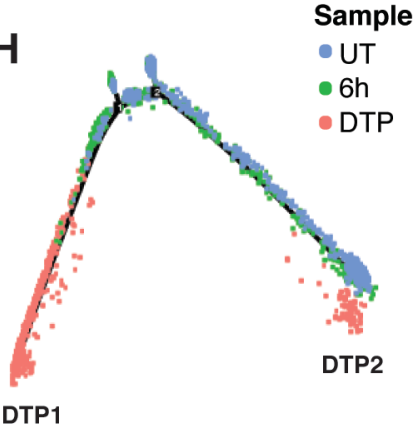

I

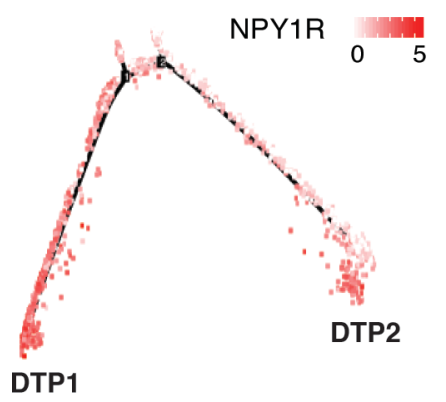

Cell Cycle

Phases

DTP1
- G0

- G1S

- G2M

- M

- MG1

- $\mathrm{S}$

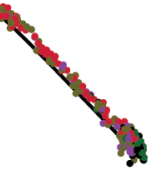

DTP2

DTP2
DTP1 
Figure 5

A

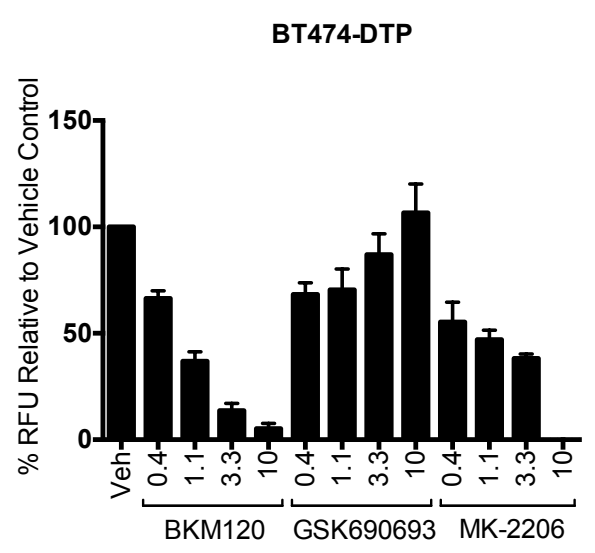

B

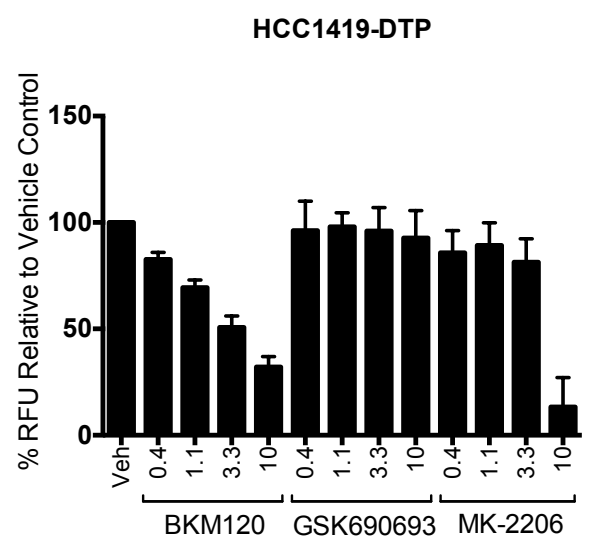

C

pPRAS40 (T246)

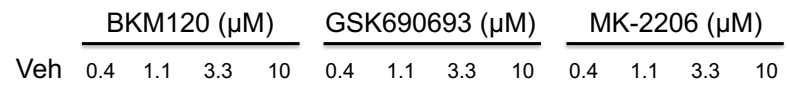

pGSK3B (S9)

\begin{tabular}{lllllllllllll|}
\hline 1.0 & 0.7 & 0.4 & 0.2 & 0.1 & 0.8 & 0.6 & 0.4 & 0.2 & 0.5 & 0.5 & 0.3 & 0.2
\end{tabular}

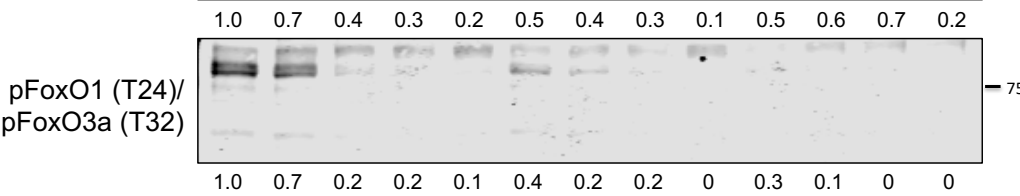

pTSC2 (S939)

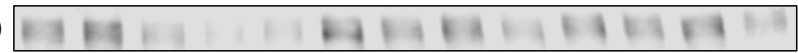

pS6K (T389) \begin{tabular}{lllllllllllll}
1.0 & 1.4 & 0.6 & 0.3 & 0.5 & 1.4 & 1.2 & 1.4 & 0.7 & 1.1 & 0.9 & 0.9 & 0.7 \\
\hline
\end{tabular}

pS6 (S240/244)

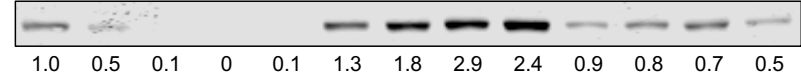

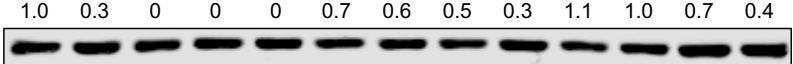

BT474-DTP

D pPRAS40 (T246)

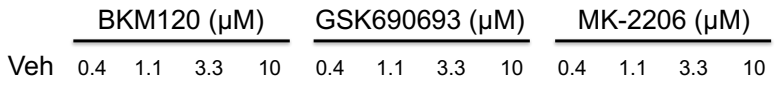
pGSK3B (S9) pFoxO1 (T24)/

pFoxO3a (T32)
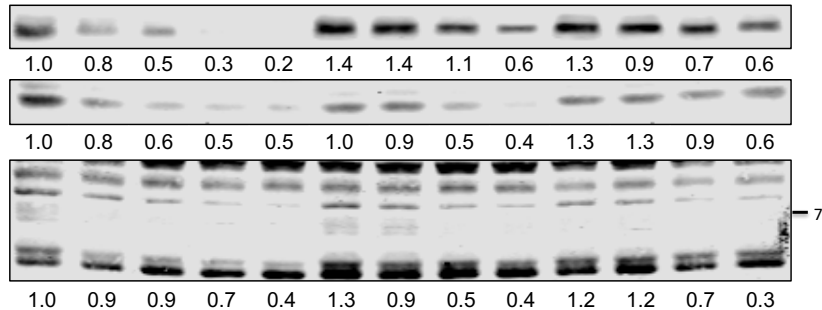

pTSC2 (S939)

pS6K (T389)

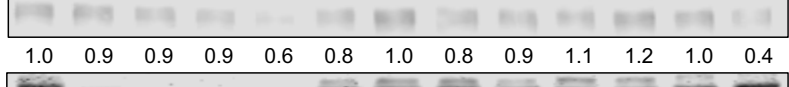

S6 (S240/244)

ERK2
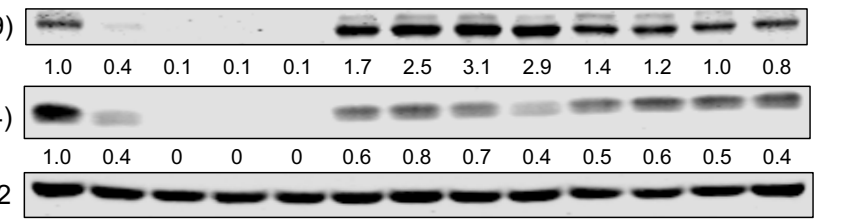

HCC1419-DTP 
A

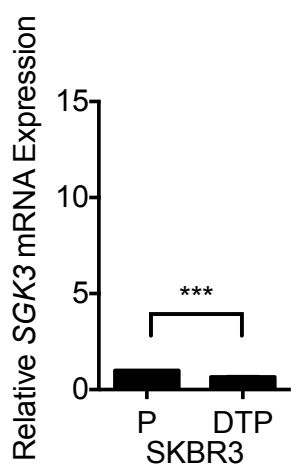

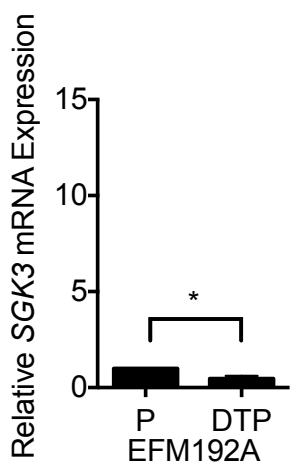
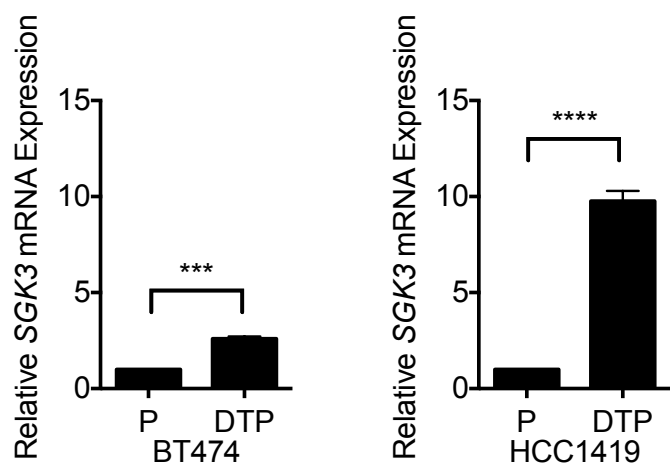

B

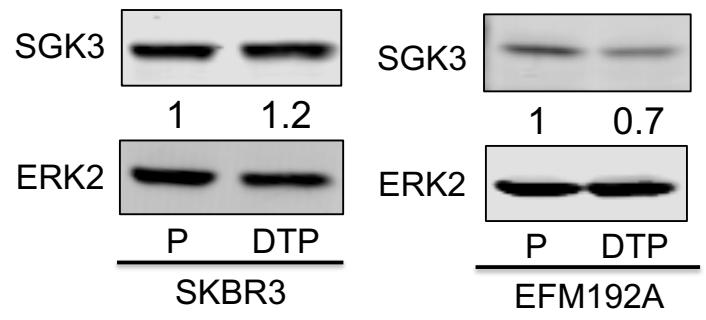

C

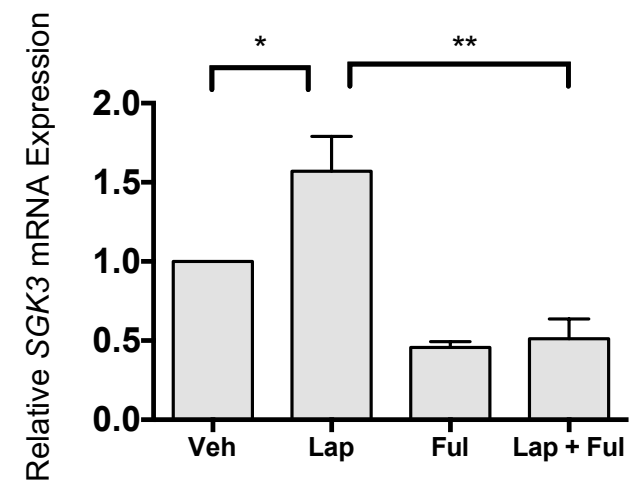

E

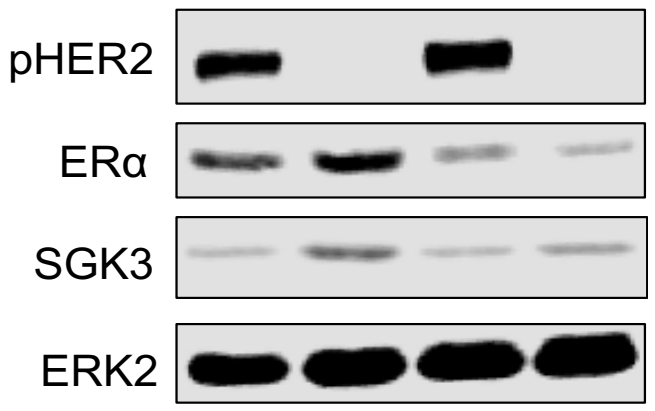

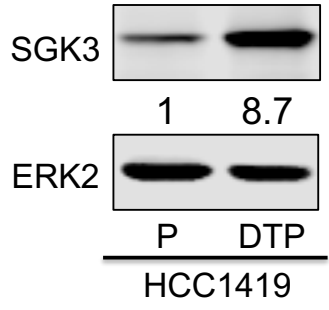

D

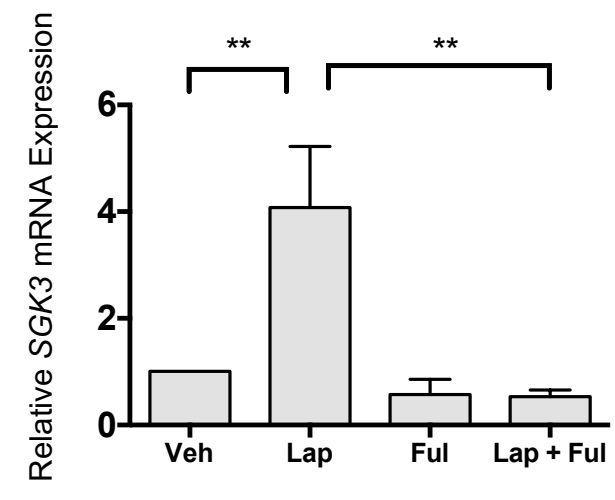

pHER2

ERa

SGK3

ERK2 


\section{A}

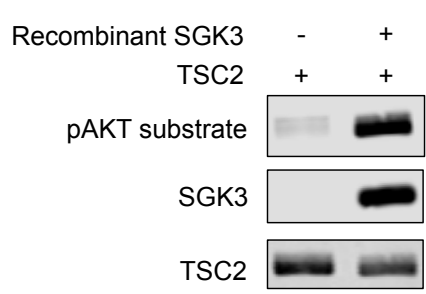

C
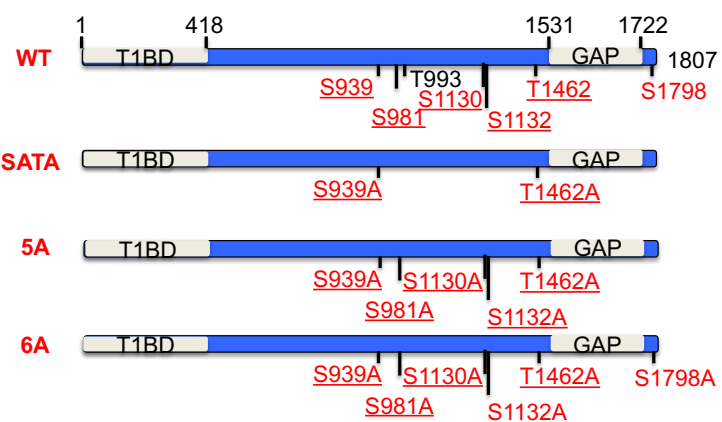

E

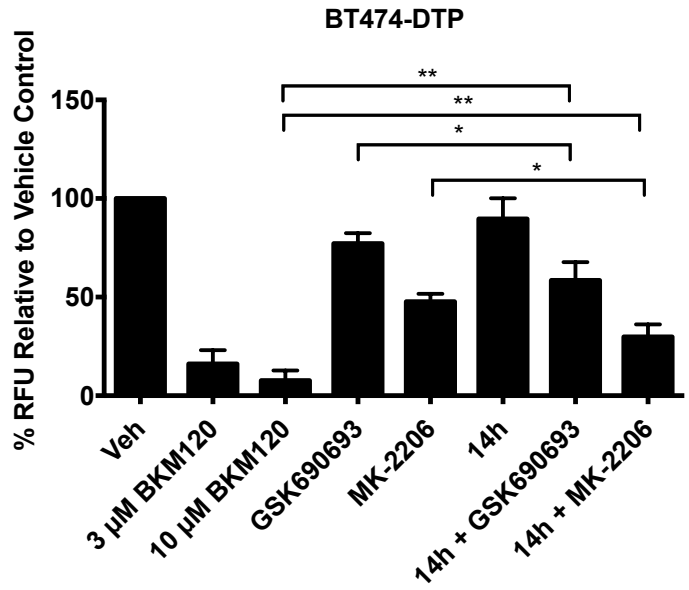

F

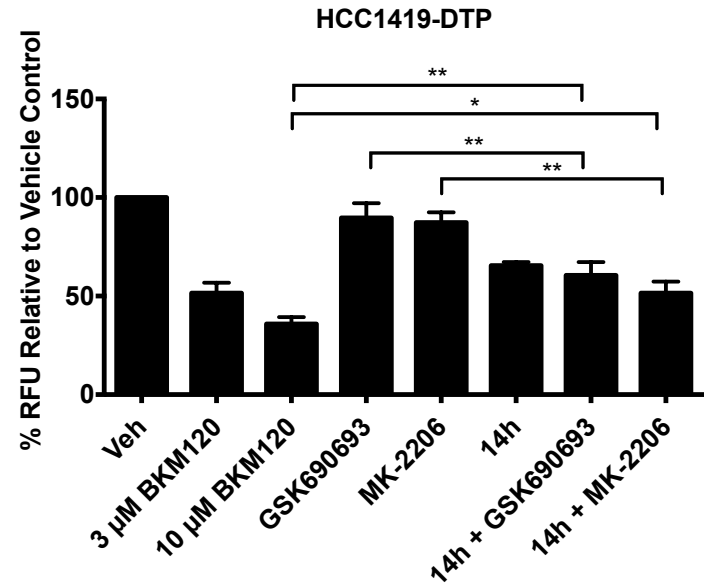

D

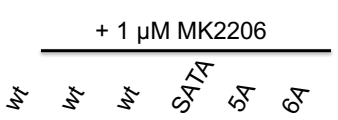

SGK3-GST

일 pAKT sub

E:a bis bs

S981 $9.0 \% \quad 47.3 \% \quad 5.3$

T993 $0.0 \% \quad 0.0 \% \quad$ N/A

$\begin{array}{llll}\mathrm{S} 1130 & 35.3 \% & 98.6 \% & 2.8\end{array}$

S1132 $50.1 \% \quad 88.4 \% \quad 1.8$

$\begin{array}{llll}\text { T1462 } & 0.2 \% & 60.7 \% & 275.9\end{array}$

2.9

$\frac{\pi}{\square}$

$\stackrel{2}{=}$

TSC2

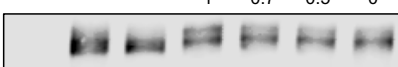

pAKT sub

TSC2

SGK3

pS6K (T389)

pS6 (S240/244)

)

pPRAS40 (T246)

ERK2

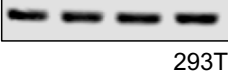

G

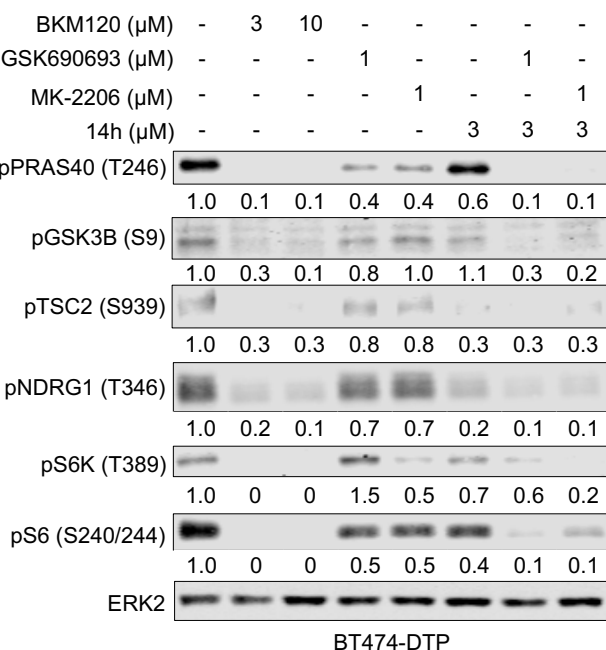

H

BT474-DTP

$\operatorname{BKM} 120(\mu \mathrm{M}) \quad-\quad 3 \quad 10$

GSK690693 $(\mu \mathrm{M})$

MK-2206 ( $\mu \mathrm{M})$

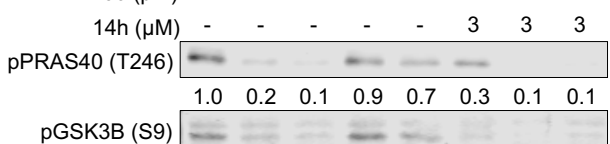

$\begin{array}{llllllll}1.0 & 0.5 & 0.6 & 1.3 & 1.1 & 0.5 & 0.3 & 0.3\end{array}$

pTSC2 (S939)

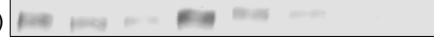

pNDRG1 (T346)

$\begin{array}{llllllll}1.0 & 0.9 & 0.6 & 1.4 & 0.7 & 0.6 & 0.4 & 0.3\end{array}$

Was

pS6K (T389)

$\begin{array}{llllllll}1.0 & 0.2 & 0.1 & 1.3 & 1.1 & 0.1 & 0 & 0\end{array}$

pS6 (S240/244)

$\begin{array}{llllllll}1.0 & 0.3 & 0.3 & 1.9 & 0.8 & 0.2 & 0.3 & 0.2\end{array}$

$\begin{array}{llllllll}1.0 & 0.1 & 0.1 & 0.9 & 0.8 & 0.1 & 0.2 & 0.1\end{array}$

ERK2 